\title{
Copper-Catalyzed Methylenation Reaction: \\ Total Synthesis of (+)-Desoxygaliellalactone
}

\author{
Hélène Lebel* and Michaël Parmentier \\ Département de chimie, Université de Montréal, Montréal, Québec, Canada, H3C 3J7 \\ Supporting Information
}

\section{Table of Contents}

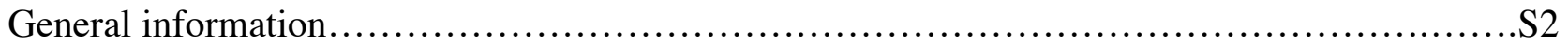

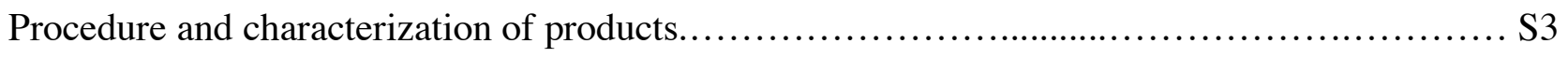

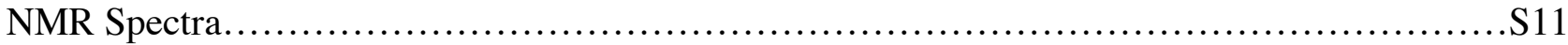


General: Unless otherwise noted, all non-aqueous reactions were performed under an oxygen-free atmosphere of argon with rigid exclusion of moisture from reagents and glassware. The solvents were dried using standard methods prior to use. 4-(tert-butyldimethylsilyloxy)butanal (12), ${ }^{1} \quad 4-$ (triisopropylsilyloxy)butanal $(\mathbf{6})^{2}$ and 4-(tert-butyldiphenylsilyloxy)butanal $(\mathbf{5})^{3}$ were prepared from 1,4-butanediol according to the literature. $\mathrm{TMSCHN}_{2}$ was prepared according to the literature (6.0-7.1 $\mathrm{M}$ as $\mathrm{TMSCHN}_{2}$; may contain traces of diethyl ether).4.5 Analytical thin layer chromatography (TLC) was performed using EM Reagent $0.25 \mathrm{~mm}$ silica gel $60-\mathrm{F}$ plates. Visualization of the developed chromatogram was performed by UV absorbance, aqueous cerium molybdate, ethanolic phosphomolybdic acid, or aqueous potassium permanganate. Flash chromatography was performed using EM Silica Gel 60 (230-400 mesh) with the indicated solvent system. Optical rotations were measured on a Perkin-Elmer 341 digital polarimeter at $589 \mathrm{~nm}$. Data are reported as follows: $[\alpha]_{1}^{\text {temp. }}$, concentration $(c \mathrm{~g} / 100 \mathrm{~mL})$, and solvent. ${ }^{1} \mathrm{H}$ NMR spectra were recorded in $\mathrm{CDCl}_{3}$, unless otherwise noted, on a Bruker AV-400, a Bruker ARX-400, a Bruker AMX-300 or a Bruker AV-300 spectrometer (400, 400, 300 and $300 \mathrm{MHz}$ respectively). Chemical shifts are reported in ppm on the $\delta$ scale from an internal standard of residual chloroform $(7.26 \mathrm{ppm})$. Data are reported as follows: chemical shift, multiplicity $(\mathrm{s}=$ singlet, $\mathrm{d}=$ doublet, $\mathrm{t}=$ triplet, $\mathrm{q}=$ quartet, $\mathrm{qn}=$ quintet, $\mathrm{m}=$ multiplet and $\mathrm{br}=$ broad $)$, coupling constant in $\mathrm{Hz}$, integration. ${ }^{13} \mathrm{C} \mathrm{NMR}$ spectra were recorded in $\mathrm{CDCl}_{3}$, unless otherwise noted, on a Bruker AV-400, a Bruker ARX-400, a Bruker AMX-300 or a Bruker AV-300 spectrometer (100, 100, 75 and $75 \mathrm{MHz}$ respectively) with complete proton decoupling. Chemical shifts are reported in ppm from the central peak of $\mathrm{CDCl}_{3}(77.0 \mathrm{ppm})$ on the $\delta$ scale. 2DCOSY experiments were realized for complex structures. Mass spectra were obtained on a LC-MSD TOF (ESI) Agilent Technologies high resolution from the Centre régional de spectrométrie de masse de l'Université de Montréal. Analytical gas chromatography with a mass spectroscopy (GC-MS) was carried out on a Hewlett Packard 6890 series gas chromatograph equipped with a split mode capillary injector and electron impact mass detector. Unless otherwise noted, injector and detector temperatures were $250{ }^{\circ} \mathrm{C}$ and the carrier gas was hydrogen $(2 \mathrm{~mL} / \mathrm{min})$ with a HP-5MS column. Data are reported as follows: column type, oven temperature, and retention time $\left(\mathrm{t}_{\mathrm{r}}\right)$.

\footnotetext{
${ }^{1}$ Kang, E. J.; Cho, E. J.; Ji, M. K.; Lee, Y. E.; Shin, D. M.; Choi, S. Y.; Chung, Y. K.; Kim, J.-S.; Kim, H.-J.; Lee, S.-G.; Lah, M. S.; Lee, E. J. Org. Chem. 2005, 70, 6321-6329.

2 Delgado, M.; Martin, J. D. J. Org. Chem. 1999, 64, 4798-4816.

${ }^{3}$ Murphy, J. A.; Rasheed, F.; Roome, S. J.; Scott, K. A.; Lewis, N. J. Chem. Soc.-Perkin Trans. 1 1998, $2331-2339$.

${ }^{4}$ Lebel, H.; Paquet, V. J. Am. Chem. Soc. 2004, 126, 320-328.

5 Although it is possible to use a commercial solution's in ether (see ref. 4), it is highly recommended to check the purity of these solutions ( ${ }^{1} \mathrm{H}$ NMR or GC-MS spectra) prior to use, as the quality change from batch to batch. For instance, we have experienced a batch from Aldrich that contain up to $50 \%$ of $\mathrm{TMSCH}_{2} \mathrm{Cl}$. See : Lebel, H.; Guay, D.; Paquet, V.; Huard, K. Org. Lett. 2004, 6, 30473050 .
} 


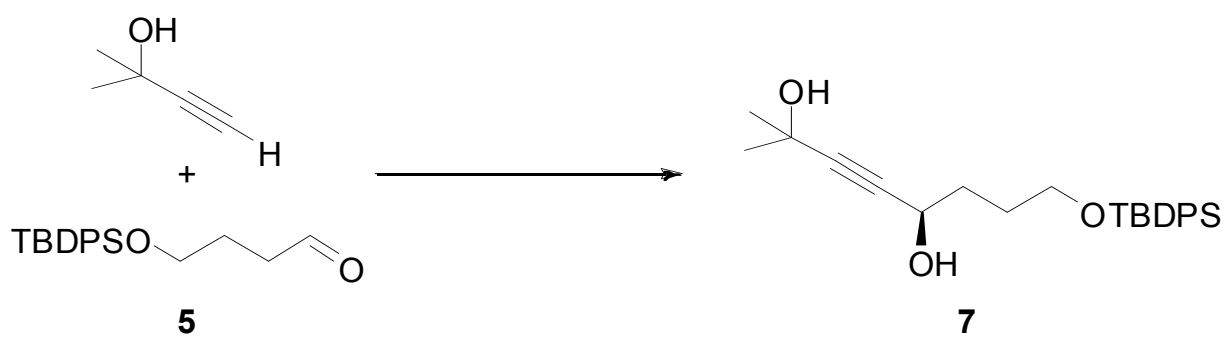

((S)-8-(tert-Butyldiphenylsilyloxy)-2-methyloct-3-yne-2,5-diol (7). To a stirred solution of $\mathrm{Zn}(\mathrm{OTf})_{2}$ $(1.09 \mathrm{~g}, 3.00 \mathrm{mmol}),(+)-N$-methylephedrine $(592 \mathrm{mg}, 3.30 \mathrm{mmol})$ in toluene $(5 \mathrm{~mL})$ under argon was added $\mathrm{Et}_{3} \mathrm{~N}(0.500 \mathrm{~mL}, 3.60 \mathrm{mmol})$. After the solution has been stirred at $23{ }^{\circ} \mathrm{C}$ for $2 \mathrm{~h}, 2$-methyl-3butyn-2-ol $(0.200 \mathrm{~mL}, 2.10 \mathrm{mmol})$ was added and the resulting mixture was stirred for $3 \mathrm{~h}$. Then, 4-(tert-butyldiphenylsilyloxy)butanal $(\mathbf{5})(326 \mathrm{mg}, 1.00 \mathrm{mmol})$ in toluene $(5 \mathrm{~mL})$ was slowly added with syringe pump over $3 \mathrm{~h}$. After $12 \mathrm{~h}$ at $23{ }^{\circ} \mathrm{C}$, the reaction mixture was quenched by addition of saturated $\mathrm{NH}_{4} \mathrm{Cl}$ solution $(5 \mathrm{~mL})$. AcOEt $(15 \mathrm{~mL})$ was then added and the two layers were separated. The aqueous layer was washed with $\operatorname{AcOEt}(3 \times 20 \mathrm{~mL})$. The combined organic layers were washed with brine $(2 \times 20 \mathrm{~mL})$, dried over $\mathrm{MgSO}_{4}$ and concentrated to give the desired diol 7 (319 $\left.\mathrm{mg}, 78 \%\right)$ as yellow oil after flash chromatography (30\% AcOEt/hexanes). $\mathrm{R}_{f} 0.11$ (30\% AcOEt/hexanes); $78 \%$ ee determined by HPLC analysis using Chiracel-OD column ( $3 \% i \mathrm{PrOH} / \mathrm{hexane}$ at $1.0 \mathrm{~mL} / \mathrm{min})$, retention time: $\mathrm{t}_{\text {major }}=22.4 \mathrm{~min}$, and $\mathrm{t}_{\text {minor }}=19.2 \mathrm{~min} ;[\alpha]_{\mathrm{D}}{ }^{25}=-8.1\left(\mathrm{c} 1.03, \mathrm{CHCl}_{3}\right) ;{ }^{1} \mathrm{H} \mathrm{NMR}(400 \mathrm{MHz}$, $\left.\mathrm{CDCl}_{3}\right) \delta$ 7.71-7.66 (m, 4H), 7.47-7.36 (m, 6H), $4.47(\mathrm{t}, J=6 \mathrm{~Hz}, 1 \mathrm{H}), 3.76-3.66(\mathrm{~m}, 2 \mathrm{H}), 2.87(\mathrm{~s}(\mathrm{br})$, $1 \mathrm{H}), 2.07$ (s (br), 1H), 1.91-1.65 (m, 4H), $1.52(\mathrm{~s}, 6 \mathrm{H}), 1.06$ (s, 9H); ${ }^{13} \mathrm{C} \mathrm{NMR}\left(100 \mathrm{MHz}, \mathrm{CDCl}_{3}\right) \delta$ 135.4, 133.3, 129.6, 127.6, 89.3, 82.9, 65.0, 63.8, 62.0, 34.9, 31.3, 28.1, 26.7, 19.0; IR (neat) 3336, 2930, 2857, 1427, $1110 \mathrm{~cm}^{-1}$; HMRS (ESI) calc. for $\mathrm{C}_{25} \mathrm{H}_{34} \mathrm{O}_{3} \mathrm{SiNa}[\mathrm{M}+\mathrm{Na}]^{+}: 433.21694$; found: 433.21666.
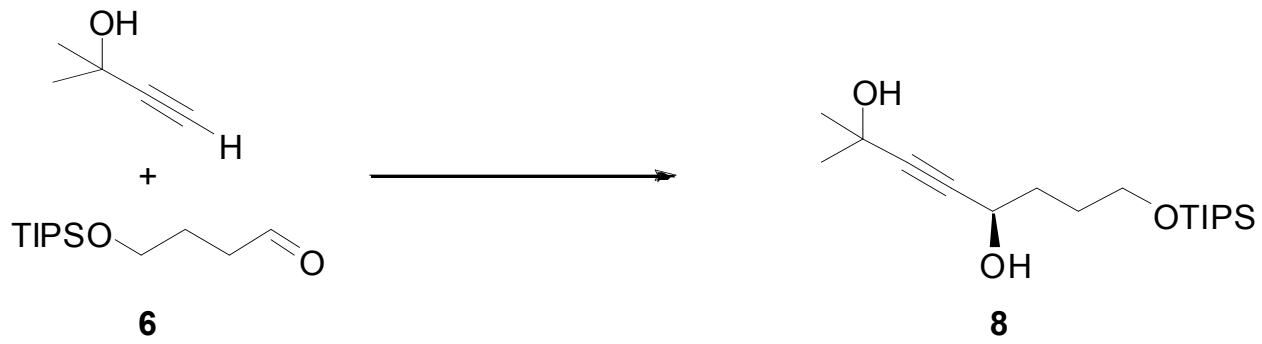

(S)-2-Methyl-8-(triisopropylsilyloxy)oct-3-yne-2,5-diol (8). To a stirred solution of $\mathrm{Zn}(\mathrm{OTf})_{2}(1.09 \mathrm{~g}$, $3.00 \mathrm{mmol}),(-)-N$-methylephedrine $(592 \mathrm{mg}, 3.30 \mathrm{mmol})$ in toluene $(5 \mathrm{~mL})$ under argon was added $\mathrm{Et}_{3} \mathrm{~N}(0.500 \mathrm{~mL}, 3.60 \mathrm{mmol})$. After the solution has been stirred at $23^{\circ} \mathrm{C}$ for $2 \mathrm{~h}, 2$-methyl-3-butyn-2ol $(0.200 \mathrm{~mL}, 2.10 \mathrm{mmol})$ was added and the resulting mixture was stirred for $3 \mathrm{~h}$. Then, 4-(triisopropylsilyloxy)butanal (6) $(244 \mathrm{mg}, 1.0 \mathrm{mmol})$ in toluene $(5 \mathrm{~mL})$ was slowly added with syringe pump over $3 \mathrm{~h}$. After $12 \mathrm{~h}$ at $23^{\circ} \mathrm{C}$, the reaction mixture was quenched by addition of saturated $\mathrm{NH}_{4} \mathrm{Cl}$ solution $(5 \mathrm{~mL})$. AcOEt $(15 \mathrm{~mL})$ was then added and the two layers were separated. The aqueous layer was washed with AcOEt $(3 \times 20 \mathrm{~mL})$. The combined organic layers were washed with brine $(2 \times 20 \mathrm{~mL})$, dried over $\mathrm{MgSO}_{4}$ and concentrated to give the desired diol $8(254 \mathrm{mg}, 77 \%)$ as colorless oil after flash chromatography (30\% AcOEt/hexanes). $\mathrm{R}_{f} 0.23$ (30\% AcOEt/hexanes); $94 \%$ ee determined by HPLC analysis of the corresponding 4-methoxybenzoate ester using Chiracel-OD column $(6 \% \mathrm{irOH} /$ hexane at $1.0 \mathrm{~mL} / \mathrm{min})$, retention time: $\mathrm{t}_{\text {major }}=11.1 \mathrm{~min}$, and $\mathrm{t}_{\text {minor }}=12.3 \mathrm{~min}$; $[\alpha]_{\mathrm{D}}{ }^{25}=-5.7\left(\mathrm{c} 0.98, \mathrm{CHCl}_{3}\right) ;{ }^{1} \mathrm{H} \mathrm{NMR}\left(400 \mathrm{MHz}, \mathrm{CDCl}_{3}\right) \delta 4.43(\mathrm{t}, J=6 \mathrm{~Hz}, 1 \mathrm{H}), 3.91$ (s (br), $\left.1 \mathrm{H}\right)$, 3.78-3.65 (m, 2H), $3.01(\mathrm{~s}(\mathrm{br}), 1 \mathrm{H}), 1.88-1.59(\mathrm{~m}, 4 \mathrm{H}), 1.48(\mathrm{~s}, 6 \mathrm{H}), 1.04(\mathrm{~m}, 21 \mathrm{H}) ;{ }^{13} \mathrm{C}$ NMR $(100$ $\left.\mathrm{MHz}, \mathrm{CDCl}_{3}\right) \delta$ 89.3, 83.0, 64.9, 63.3, 61.9, 35.1, 31.3, 28.7, 17.9, 11.8; IR (neat) 3326, 2941, 2865, 
1462, 1364, 1102, $881 \mathrm{~cm}^{-1}$; HMRS (ESI) calc. for $\mathrm{C}_{18} \mathrm{H}_{36} \mathrm{O}_{3} \mathrm{SiNa}[\mathrm{M}+\mathrm{Na}]^{+}$: 351.23259; found: 351.23284 .
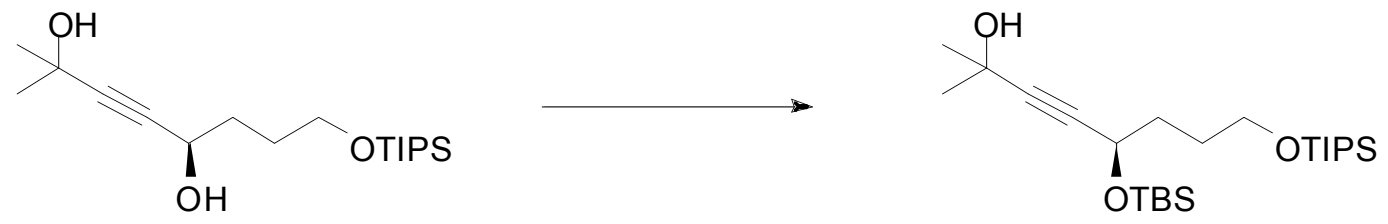

8

5-(tert-Butyldimethylsilyloxy)-2-methyl-8-(triisopropylsilyloxy)oct-3-yn-2-ol. To a solution of diol 8 (25.0 g, $76.3 \mathrm{mmol})$ in $\mathrm{CH}_{2} \mathrm{Cl}_{2}(700 \mathrm{~mL})$, was added imidazole $(13.3 \mathrm{~g}, 91.6 \mathrm{mmol})$ and DMAP (2.80 g, $22.9 \mathrm{mmol})$. A solution of TBSCl $(14.1 \mathrm{~g}, 91.6 \mathrm{mmol})$ in $\mathrm{CH}_{2} \mathrm{Cl}_{2}(60 \mathrm{~mL})$ was added drop wise at $23{ }^{\circ} \mathrm{C}$ and the resulting mixture was stirred for $12 \mathrm{~h}$. The reaction mixture was quenched by addition of saturated aqueous $\mathrm{NH}_{4} \mathrm{Cl}(200 \mathrm{~mL})$. The two layers were separated and the aqueous layer was washed with $\mathrm{CH}_{2} \mathrm{Cl}_{2}(3 \times 400 \mathrm{~mL})$. The combined organic layers were washed with $\mathrm{HCl} 10 \%(2 \times 100 \mathrm{~mL})$ and brine $(100 \mathrm{~mL})$, dried over $\mathrm{MgSO}_{4}$ and concentrated to give the desired product $(27.9 \mathrm{~g}, 82 \%)$ as colorless oil after flash chromatography (10\% AcOEt/hexanes). $\mathrm{R}_{f} 0.28$ (10\% AcOEt/hexanes); ${ }^{1} \mathrm{H}$ NMR $\left(300 \mathrm{MHz}, \mathrm{C}_{6} \mathrm{D}_{6}\right) \delta 4.48(\mathrm{t}, J=6 \mathrm{~Hz}, 1 \mathrm{H}), 3.66(\mathrm{t}, J=6 \mathrm{~Hz}, 2 \mathrm{H}), 1.98-1.72(\mathrm{~m}, 4 \mathrm{H}), 1.51(\mathrm{~s}$, $1 \mathrm{H}), 1.39(\mathrm{~s}, 6 \mathrm{H}), 1.14-1.07(\mathrm{~m}, 21 \mathrm{H}), 1.02(\mathrm{~s}, 9 \mathrm{H}), 0.24(\mathrm{~s}, 3 \mathrm{H}), 0.17(\mathrm{~s}, 3 \mathrm{H}) ;{ }^{13} \mathrm{C} \mathrm{NMR}(75 \mathrm{MHz}$, $\left.\mathrm{C}_{6} \mathrm{D}_{6}\right) \delta 89.7,83.7,64.8,63.4,63.3,35.8,31.6,31.5,29.3,26.0,18.3,12.3,-4.0,-4.8$; IR (neat) 3363, 2941, 2865, 1751, 1463, 1252, 1102, $835 \mathrm{~cm}^{-1}$; HMRS (ESI) calc. for $\mathrm{C}_{24} \mathrm{H}_{50} \mathrm{O}_{3} \mathrm{Si}_{2} \mathrm{Na}[\mathrm{M}+\mathrm{Na}]^{+}$: 465.31907; found: 465.31807.
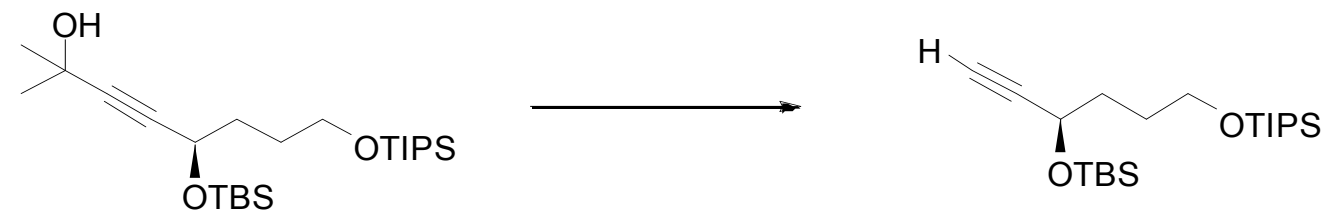

9

3-(tert-Butyldimethylsilyloxy)-6-(triisopropylsilyloxy)-1-hexyne (9). A flask was charged with ground potassium tert-butoxide $(8.85 \mathrm{~g}, 78.9 \mathrm{mmol})$ and 18-crown-6 (800 $\mathrm{mg}, 3.00 \mathrm{mmol})$ and then purged with argon. Toluene $(300 \mathrm{~mL})$ was added followed by the alkyne $(27.0 \mathrm{~g}, 60.7 \mathrm{mmol})$. The resulting suspension was heated at $45^{\circ} \mathrm{C}$ under a stream of argon for 2 hours. The reaction mixture was quenched by addition of saturated aqueous $\mathrm{NH}_{4} \mathrm{Cl}(60 \mathrm{~mL})$. The two layers were separated and the aqueous layer was washed with $\operatorname{AcOEt}(3 \times 150 \mathrm{~mL})$. The combined organic layers were washed with brine $(2 \times 70 \mathrm{~mL})$, dried over $\mathrm{MgSO}_{4}$ and concentrated in vacuo to give the desired product 9 (16.4 g, $70 \%)$ as colorless oil after flash chromatography (2\% AcOEt/hexanes). $\mathrm{R}_{f} 0.81$ (5\% AcOEt/hexanes); ${ }^{1} \mathrm{H}$ NMR (400 MHz, $\left.\mathrm{C}_{6} \mathrm{D}_{6}\right) \delta 4.41(\mathrm{dt}, J=6,2 \mathrm{~Hz}, 1 \mathrm{H}), 3.62(\mathrm{t}, J=6 \mathrm{~Hz}, 2 \mathrm{H}), 2.03(\mathrm{~d}, J=2 \mathrm{~Hz}, 1 \mathrm{H})$, 1.94-1.84 (m, 2H), 1.84-1.72 (m, 2H), 1.13-1.07 (m, 21H), $1.00(\mathrm{~s}, 9 \mathrm{H}), 0.22(\mathrm{~s}, 3 \mathrm{H}), 0.14(\mathrm{~s}, 3 \mathrm{H}) ;{ }^{13} \mathrm{C}$ NMR $\left(100 \mathrm{MHz}, \mathrm{C}_{6} \mathrm{D}_{6}\right) \delta 85.7,72.6,63.3,63.1,35.6,29.1,26.0,18.3,12.3,-4.3$, -4.9; IR (neat) 2943, 2866, 2094, 1686, 1464, 1255, 1100, 1024, $882 \mathrm{~cm}^{-1}$; HMRS (ESI) calc. for $\mathrm{C}_{21} \mathrm{H}_{44} \mathrm{O}_{2} \mathrm{Si}_{2} \mathrm{Na}[\mathrm{M}+\mathrm{Na}]^{+}$: 407.27720; found: 407.27686. 


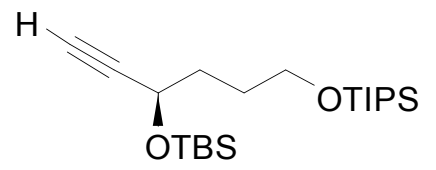

9

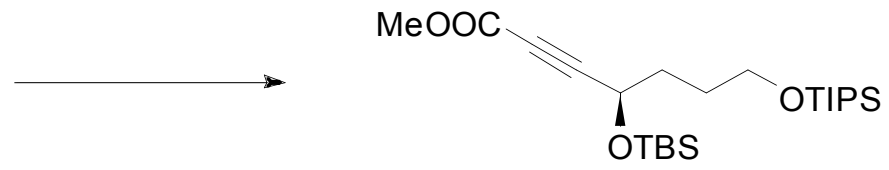

10

Methyl 4-(tert-butyldimethylsilyloxy)-7-(triisopropylsilyloxy)hept-2-ynoate (10). To a solution of alkyne 9 (7.78 g, $20.1 \mathrm{mmol})$ in THF (200 mL) at $0{ }^{\circ} \mathrm{C}$, was slowly added $n$-BuLi (2.5 $\mathrm{M}$ in $n$-hexane) $(9.66 \mathrm{~mL}, 24.1 \mathrm{mmol})$, and the resulting mixture was stirred for 2 hours. The mixture was then cooled to $-78{ }^{\circ} \mathrm{C}$, and methyl chloroformate $(2.17 \mathrm{~mL}, 28.1 \mathrm{mmol})$ was added drop wise. After $30 \mathrm{~min}$, the temperature was warmed to $23{ }^{\circ} \mathrm{C}$ and the solution was quenched with saturated $\mathrm{NH}_{4} \mathrm{Cl}(70 \mathrm{~mL})$. The two layers were separated and the aqueous layer was washed with AcOEt (3 x $100 \mathrm{~mL})$. The combined organic layers were washed with brine $(70 \mathrm{~mL})$, dried over $\mathrm{MgSO}_{4}$ and concentrated in vacuo to give the desired product $\mathbf{1 0}(7.72 \mathrm{~g}, 86 \%)$ as colorless oil after flash chromatography (3\% AcOEt/hexanes). $\mathrm{R}_{f} 0.43$ (5\% AcOEt/hexanes); ${ }^{1} \mathrm{H}$ NMR $\left(300 \mathrm{MHz}, \mathrm{C}_{6} \mathrm{D}_{6}\right) \delta 4.39(\mathrm{t}, J=6 \mathrm{~Hz}, 1 \mathrm{H}), 3.56(\mathrm{t}, J=6 \mathrm{~Hz}$, $2 \mathrm{H}), 3.21(\mathrm{~s}, 3 \mathrm{H}), 1.90-1.60(\mathrm{~m}, 4 \mathrm{H}), 1.15-1.05(\mathrm{~m}, 21 \mathrm{H}), 0.96(\mathrm{~s}, 9 \mathrm{H}), 0.20(\mathrm{~s}, 3 \mathrm{H}), 0.10(\mathrm{~s}, 3 \mathrm{H})$; ${ }^{13} \mathrm{C}$ NMR $\left(75 \mathrm{MHz}, \mathrm{C}_{6} \mathrm{D}_{6}\right) \delta 153.7,88.7,76.9,63.1,63.0,52.0,34.8,28.8,25.9,18.2,12.3,-4.4,-5.1$; IR (neat) 3397, 2929, 2866, 1721, 1463, 1255, 1096, $882 \mathrm{~cm}^{-1}$; HMRS (ESI) calc. for $\mathrm{C}_{23} \mathrm{H}_{46} \mathrm{O}_{4} \mathrm{Si}_{2} \mathrm{Na}$ $[\mathrm{M}+\mathrm{Na}]^{+}:$465.28268; found: 465.28182 .

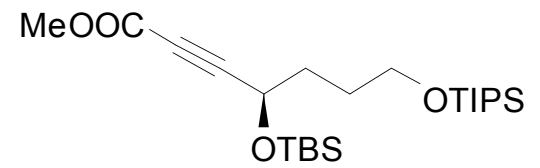

10

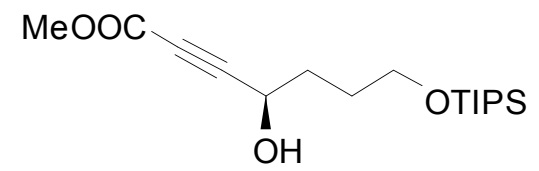

11

Methyl 4-hydroxy-7-(triisopropylsilyloxy)hept-2-ynoate (11). To a solution of 10 (2.21 g, 3.00 $\mathrm{mmol})$ in $\mathrm{CH}_{2} \mathrm{Cl}_{2}(30 \mathrm{~mL})$ at $-50{ }^{\circ} \mathrm{C}$, was added drop wise TMSOTf $(0.60 \mathrm{~mL}, 3.3 \mathrm{mmol})$ and the resulting mixture was stirred 3 hours. The reaction was quenched by addition of saturated aqueous $\mathrm{NH}_{4} \mathrm{Cl}(20 \mathrm{~mL})$. The two layers were separated and the aqueous layer was washed with $\mathrm{CH}_{2} \mathrm{Cl}_{2}(3 \times 50$ $\mathrm{mL})$. The combined organic layers were washed with brine $(50 \mathrm{~mL})$, dried over $\mathrm{MgSO}_{4}$ and concentrated to give the desired product $11(1.68 \mathrm{~g}, 57 \%)$ as a colorless oil after flash chromatography (15\% AcOEt/hexanes). $\mathrm{R}_{f} 0.28$ (20\% AcOEt/hexanes); ${ }^{1} \mathrm{H} \mathrm{NMR}\left(400 \mathrm{MHz}, \mathrm{C}_{6} \mathrm{D}_{6}\right) \delta 4.18(\mathrm{q}, J=6 \mathrm{~Hz}$, $1 \mathrm{H}), 3.57-3.33(\mathrm{~m}, 2 \mathrm{H}), 3.24(\mathrm{~s}, 3 \mathrm{H}), 2.53(\mathrm{~d}, J=6 \mathrm{~Hz}, 1 \mathrm{H}), 1.70-1.42(\mathrm{~m}, 4 \mathrm{H}), 1.11-1.02(\mathrm{~m}, 21 \mathrm{H})$; ${ }^{13} \mathrm{C}$ NMR $\left(100 \mathrm{MHz}, \mathrm{C}_{6} \mathrm{D}_{6}\right) \delta 153.9,88.8,76.8,63.3,61.8,52.0,34.4,28.6,18.2,12.2$; IR (neat) 3404, 2944, 2866, 2237, 1720, 1435, 1252, $1107 \mathrm{~cm}^{-1}$; HMRS (ESI) calc. for $\mathrm{C}_{17} \mathrm{H}_{32} \mathrm{O}_{4} \mathrm{SiNa}[\mathrm{M}+\mathrm{Na}]^{+}$: 351.1974; found: 351.1967.

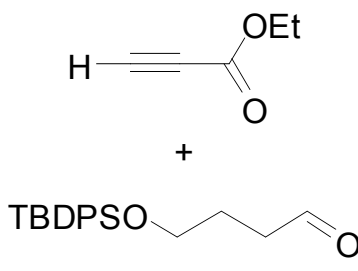

5

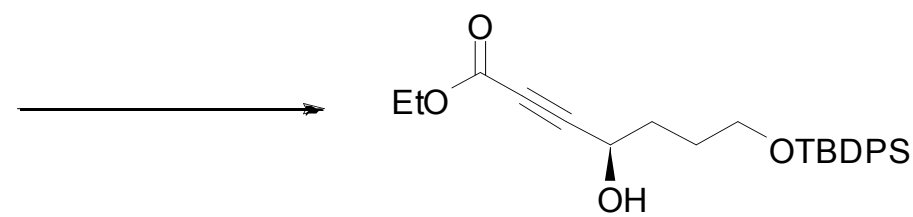

13

(R)-Ethyl 7-(tert-butyldiphenylsilyloxy)-4-hydroxyhept-2-ynoate (13). To a stirred solution of $(S)$ BINOL (114 mg, $0.400 \mathrm{mmol})$, HMPA $(348 \mu \mathrm{L}, 2.00 \mathrm{mmol})$, ethyl propiolate $(0.40 \mathrm{~mL}, 4.0 \mathrm{mmol})$ in $\mathrm{CH}_{2} \mathrm{Cl}_{2}(12 \mathrm{~mL})$ under argon was slowly added $\mathrm{Et}_{2} \mathrm{Zn}(0.41 \mathrm{~mL}, 4.0 \mathrm{mmol})$. After the solution has been 
stirred at $23{ }^{\circ} \mathrm{C}$ for $16 \mathrm{~h}, \operatorname{Ti}(\mathrm{OiPr})_{4}(296 \mu \mathrm{L}, 1.00 \mathrm{mmol})$ was added and the stirring was continued for another hour. The aldehyde (5) $(326 \mathrm{mg}, 1.00 \mathrm{mmol})$ in $\mathrm{CH}_{2} \mathrm{Cl}_{2}(2 \mathrm{~mL})$ was then slowly added with syringe pump over $4 \mathrm{~h}$. After another $4 \mathrm{~h}$ at $23{ }^{\circ} \mathrm{C}$, the reaction mixture was quenched by addition of $1 \mathrm{M}$ aqueous Rochelle's salt and stirred for $30 \mathrm{~min}$. Aqueous solution of $\mathrm{NH}_{4} \mathrm{Cl}(10 \mathrm{~mL})$ and $1 \mathrm{M} \mathrm{HCl}(2$ $\mathrm{mL})$ solution were then added and the two layers were separated. The aqueous layer was washed with $\mathrm{Et}_{2} \mathrm{O}(3 \times 40 \mathrm{~mL})$. The combined organic layers were washed with brine $(2 \times 20 \mathrm{~mL})$, dried over $\mathrm{MgSO}_{4}$ and concentrated to give the desired alcohol $13(254 \mathrm{mg}, 60 \%)$ as a colorless oil after flash chromatography (20\% AcOEt/hexanes). $\mathrm{R}_{f} 0.17$ (15\% AcOEt/hexanes); $77 \%$ ee determined by HPLC analysis using Chiracel-OD column $(5 \% \mathrm{PrOH} / \mathrm{hexane}$ at $1.0 \mathrm{~mL} / \mathrm{min})$, retention time: $\mathrm{t}_{\text {major }}=13.2$ $\min$, and $\mathrm{t}_{\text {minor }}=11.8 \mathrm{~min} ;[\alpha]_{\mathrm{D}}{ }^{25}=-14.2\left(\mathrm{c} 0.50, \mathrm{CHCl}_{3}\right) ;{ }^{1} \mathrm{H} \mathrm{NMR}\left(300 \mathrm{MHz}, \mathrm{CDCl}_{3}\right) \delta 7.72-7.63(\mathrm{~m}$, $4 \mathrm{H}), 7.48-7.35(\mathrm{~m}, 6 \mathrm{H}), 4.58(\mathrm{q}, J=6 \mathrm{~Hz}, 1 \mathrm{H}), 4.24(\mathrm{q}, J=7 \mathrm{~Hz}, 2 \mathrm{H}), 3.80-3.60(\mathrm{~m}, 2 \mathrm{H}), 3.27(\mathrm{~d}, J=6$ $\mathrm{Hz}, 1 \mathrm{H}), 2.02-1.61(\mathrm{~m}, 4 \mathrm{H}), 1.31(\mathrm{t}, J=7 \mathrm{~Hz}, 3 \mathrm{H}), 1.06(\mathrm{~s}, 9 \mathrm{H}) ;{ }^{13} \mathrm{C}$ NMR $\left(75 \mathrm{MHz}, \mathrm{DMSO}-d_{6}\right) \delta$ $152.4,134.5,132.8,129.4,127.4,89.5,74.8,62.7,61.4,59.6,32.7,27.3,26.2,18.3,13.3$; IR (neat) 3405, 2931, 2857, 2235, 1711, 1472, 1427, 1389, 1366, 1243, 1105, $1008 \mathrm{~cm}^{-1}$; HMRS (ESI) calc. for $\mathrm{C}_{25} \mathrm{H}_{32} \mathrm{O}_{4} \mathrm{SiNa}[\mathrm{M}+\mathrm{Na}]^{+}$: 447.19621; found: 447.19627.

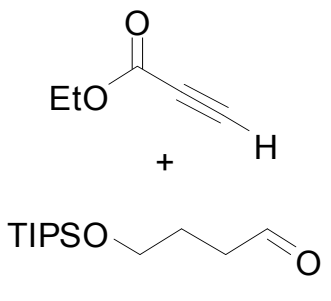

6

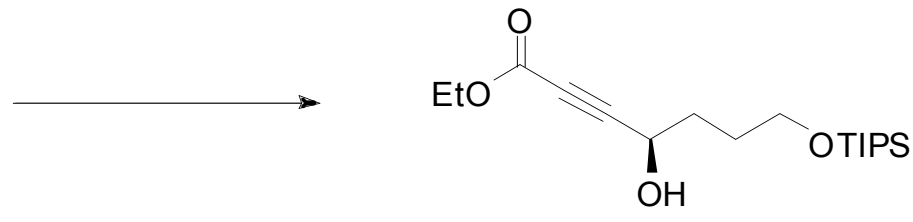

14

(R)-Ethyl 4-hydroxy-7-(triisopropylsilyloxy)hept-2-ynoate (14). To a stirred solution of $(S)$-BINOL (114 mg, $0.400 \mathrm{mmol}), \mathrm{HMPA}(348 \mu \mathrm{L}, 2.00 \mathrm{mmol})$, ethyl propiolate $(0.40 \mathrm{~mL}, 4.0 \mathrm{mmol})$ in $\mathrm{CH}_{2} \mathrm{Cl}_{2}$ $(12 \mathrm{~mL})$ under argon was slowly added $\mathrm{Et}_{2} \mathrm{Zn}(0.41 \mathrm{~mL}, 4.0 \mathrm{mmol})$. After the solution has been stirred at $23{ }^{\circ} \mathrm{C}$ for $16 \mathrm{~h}, \mathrm{Ti}(\mathrm{OiPr})_{4}(296 \mu \mathrm{L}, 1.00 \mathrm{mmol})$ was added and the stirring was continued for another hour. The aldehyde (6) $(244 \mathrm{mg}, 1.00 \mathrm{mmol})$ in $\mathrm{CH}_{2} \mathrm{Cl}_{2}(2 \mathrm{~mL})$ was then slowly added with syringe pump over $4 \mathrm{~h}$. After another $4 \mathrm{~h}$ at $23{ }^{\circ} \mathrm{C}$, the reaction mixture was quenched by addition of $1 \mathrm{M}$ aqueous Rochelle's salt and stirred for $30 \mathrm{~min}$. Aqueous solution of $\mathrm{NH}_{4} \mathrm{Cl}(10 \mathrm{~mL})$ and $1 \mathrm{M} \mathrm{HCl}(2$ $\mathrm{mL})$ solution were then added and the two layers were separated. The aqueous layer was washed with $\mathrm{Et}_{2} \mathrm{O}(3 \times 40 \mathrm{~mL})$. The combined organic layers were washed with brine $(2 \times 20 \mathrm{~mL})$, dried over $\mathrm{MgSO}_{4}$ and concentrated to give the desired alcohol 14 (192 mg, 64\%) as a pale yellow oil after flash chromatography (20\% AcOEt/hexanes). $\mathrm{R}_{f} 0.11$ (10\% AcOEt/hexanes); 88\% ee determined by HPLC analysis using Chiracel-OD column $(5 \% \mathrm{iPrOH} /$ hexane at $1.0 \mathrm{~mL} / \mathrm{min})$, retention time: $\mathrm{t}_{\text {major }}=7.3 \mathrm{~min}$, and $\mathrm{t}_{\text {minor }}=6.4 \mathrm{~min} ;[\alpha]_{\mathrm{D}}{ }^{25}=-22.3\left(\mathrm{c} 0.52, \mathrm{CHCl}_{3}\right) ;{ }^{1} \mathrm{H} \mathrm{NMR}\left(400 \mathrm{MHz}, \mathrm{CDCl}_{3}\right) \delta 4.63-4.53(\mathrm{~m}, 1 \mathrm{H})$, $4.23(\mathrm{q}, J=7 \mathrm{~Hz}, 2 \mathrm{H}), 3.97(\mathrm{~d}, J=7 \mathrm{~Hz}, 1 \mathrm{H}), 3.86-3.78(\mathrm{~m}, 1 \mathrm{H}), 3.77-3.70(\mathrm{~m}, 1 \mathrm{H}), 1.98-1.81(\mathrm{~m}$, $3 \mathrm{H}), 1.80-1.68(\mathrm{~m}, 1 \mathrm{H}), 1.30(\mathrm{t}, J=7 \mathrm{~Hz}, 3 \mathrm{H}), 1.08(\mathrm{~m}, 21 \mathrm{H}) ;{ }^{13} \mathrm{C}$ NMR $\left(75 \mathrm{MHz}, \mathrm{CDCl}_{3}\right) \delta 153.4$, 88.0, 76.3, 63.3, 61.9, 61.6, 34.7, 28.4, 17.8, 13.9, 11.8; IR (neat) 3385, 2942, 2865, 1714, 1463, 1243, 1104, $882 \mathrm{~cm}^{-1}$; HMRS (ESI) calc. for $\mathrm{C}_{18} \mathrm{H}_{34} \mathrm{O}_{4} \mathrm{SiNa}[\mathrm{M}+\mathrm{Na}]^{+}:$365.21186; found: 365.21153. 


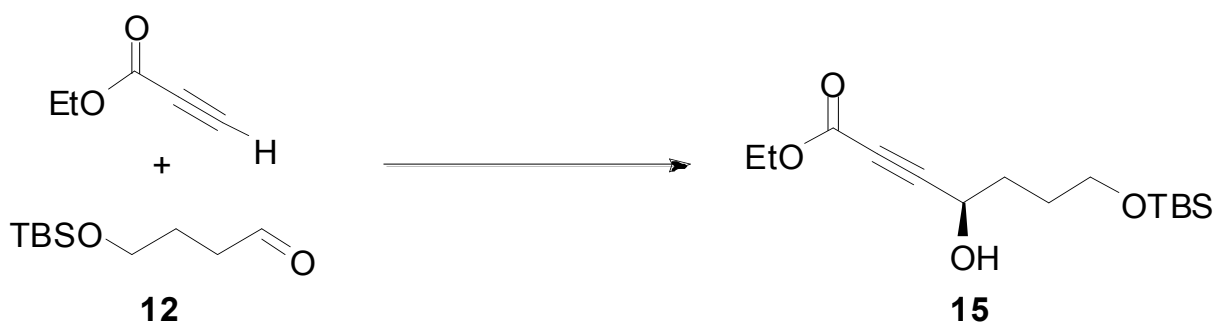

(R)-Ethyl 7-(tert-butyldimethylsilyloxy)-4-hydroxyhept-2-ynoate (15). To a stirred solution of $(S)$ BINOL (114 mg, $0.400 \mathrm{mmol})$, HMPA $(348 \mu \mathrm{L}, 2.00 \mathrm{mmol})$, ethyl propiolate $(0.4 \mathrm{~mL}, 4.0 \mathrm{mmol})$ in $\mathrm{CH}_{2} \mathrm{Cl}_{2}(12 \mathrm{~mL})$ under argon was slowly added $\mathrm{Et}_{2} \mathrm{Zn}(0.41 \mathrm{~mL}, 4.0 \mathrm{mmol})$. After the solution has been stirred at $23{ }^{\circ} \mathrm{C}$ for $16 \mathrm{~h}, \mathrm{Ti}(\mathrm{OiPr})_{4}(296 \mu \mathrm{L}, 1.0 \mathrm{mmol})$ was added and the stirring was continued for another hour. The aldehyde (12) $(202 \mathrm{mg}, 1.00 \mathrm{mmol})$ in $\mathrm{CH}_{2} \mathrm{Cl}_{2}(2 \mathrm{~mL})$ was then slowly added with syringe pump over $4 \mathrm{~h}$. After another $4 \mathrm{~h}$ at $23{ }^{\circ} \mathrm{C}$, the reaction mixture was quenched by addition of $1 \mathrm{M}$ aqueous Rochelle's salt and stirred for $30 \mathrm{~min}$. Aqueous solution of $\mathrm{NH}_{4} \mathrm{Cl}(10 \mathrm{~mL})$ and $1 \mathrm{M} \mathrm{HCl}(2$ $\mathrm{mL}$ ) solution were then added and the two layers were separated. The aqueous layer was washed with $\mathrm{Et}_{2} \mathrm{O}(3 \times 40 \mathrm{~mL})$. The combined organic layers were washed with brine $(2 \times 20 \mathrm{~mL})$, dried over $\mathrm{MgSO}_{4}$ and concentrated to give the desired alcohol desired alcohol 15 (176 $\left.\mathrm{mg}, 87 \%\right)$ as a pale yellow oil after flash chromatography (20\% AcOEt/hexanes). $\mathrm{R}_{f} 0.11$ (10\% AcOEt/hexanes); $90 \%$ ee determined by HPLC analysis using Chiracel-OD column $(5 \% \mathrm{iPrOH} / \mathrm{hexane}$ at $1.0 \mathrm{~mL} / \mathrm{min})$, retention time: $\mathrm{t}_{\text {major }}=7.6 \mathrm{~min}$, and $\mathrm{t}_{\text {minor }}=6.8 \mathrm{~min} ;[\alpha]_{\mathrm{D}}{ }^{25}=-19.2\left(\mathrm{c} 1.00, \mathrm{CHCl}_{3}\right) ;{ }^{1} \mathrm{H} \mathrm{NMR}(400 \mathrm{MHz}$, $\left.\mathrm{CDCl}_{3}\right) \delta 4.63-4.54(\mathrm{~m}, 1 \mathrm{H}), 4.25(\mathrm{q}, J=7 \mathrm{~Hz}, 2 \mathrm{H}), 4.01(\mathrm{~d}, J=7 \mathrm{~Hz}, 1 \mathrm{H}), 3.80-3.73(\mathrm{~m}, 1 \mathrm{H}), 3.71-$ $3.63(\mathrm{~m}, 1 \mathrm{H}), 2.02-1.80(\mathrm{~m}, 3 \mathrm{H}), 1.79-1.69(\mathrm{~m}, 1 \mathrm{H}), 1.32(\mathrm{t}, J=7 \mathrm{~Hz}, 3 \mathrm{H}), 0.93(\mathrm{~s}, 9 \mathrm{H}), 0.12(\mathrm{~s}, 3 \mathrm{H})$, $0.11(\mathrm{~s}, 3 \mathrm{H}) ;{ }^{13} \mathrm{C}$ NMR $\left(100 \mathrm{MHz}, \mathrm{DMSO}-d_{6}\right) \delta 152.8,89.9,75.2,62.1,61.8,60.0,33.2,28.0,25.8$, 17.9, 13.8, -5.3; IR (neat) 3405, 2930, 2858, 1714, $1247 \mathrm{~cm}^{-1}$; HMRS (ESI) calc. for $\mathrm{C}_{15} \mathrm{H}_{29} \mathrm{O}_{4} \mathrm{Si}$ $[\mathrm{M}+\mathrm{H}]^{+}:$301.1829; found: 301.1834 .

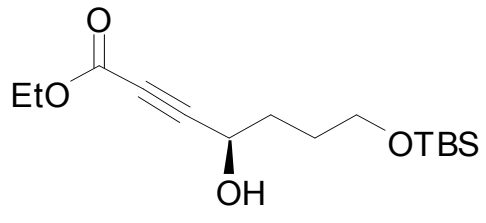

15

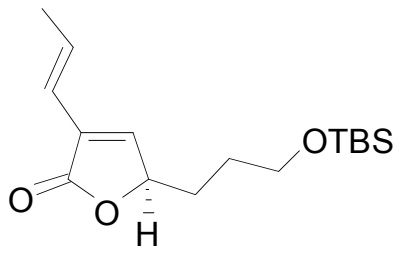

16

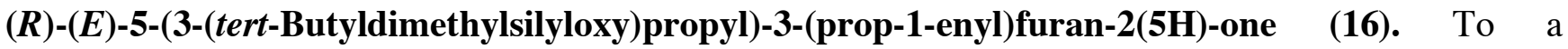
solution of $\mathrm{Pd}\left(\mathrm{PPh}_{3}\right)_{4}(2.89 \mathrm{~g}, 2.50 \mathrm{mmol})$ in $\mathrm{THF}(500 \mathrm{~mL})$ at $0{ }^{\circ} \mathrm{C}$, was added the ester $15(15.2 \mathrm{~g}$, $50.0 \mathrm{mmol})$. After $10 \mathrm{~min}, \mathrm{Bu}_{3} \mathrm{SnH}(13.5 \mathrm{~mL}, 51.0 \mathrm{mmol})$ was then added drop wise and the resulting mixture was stirred $1 \mathrm{~h}$ at $0{ }^{\circ} \mathrm{C}$. CuI $(1.90 \mathrm{~g}, 10.0 \mathrm{mmol}), \mathrm{CsF}(9.11 \mathrm{~g}, 60.0 \mathrm{mmol})$ then $1-$ Bromopropene (cis/trans mixture) $(8.56 \mathrm{~mL}, 100 \mathrm{mmol})$ were added sequentially. The reaction mixture was stirred for $12 \mathrm{~h}$ at $23{ }^{\circ} \mathrm{C}$. Water $(100 \mathrm{~mL})$ was added, the mixture was filtered and the solid was washed with a 1:1 mixture of AcOEt: $\mathrm{CH}_{2} \mathrm{Cl}_{2}(200 \mathrm{~mL})$. The two layers were separated and the aqueous layer was washed with $\mathrm{CH}_{2} \mathrm{Cl}_{2}(3 \times 100 \mathrm{~mL})$. The combined organic layers were washed with brine $(100 \mathrm{~mL})$, dried over $\mathrm{MgSO}_{4}$ and concentrated in vacuo to give $10.79 \mathrm{~g} \mathrm{(72 \% )}$ of a cis/trans mixture of 16 as a pale yellow oil after flash chromatography (15\% $\mathrm{Et}_{2} \mathrm{O} /$ pentane). $\mathrm{R}_{f} 0.26$ and 0.25 (15\% $\mathrm{Et}_{2} \mathrm{O}$ /pentane). The mixture was dissolved in $\mathrm{CCl}_{4}(100 \mathrm{~mL})$ and iodine $(2.53 \mathrm{~g}, 10.0 \mathrm{mmol})$ was added to the solution. The mixture was then irradiated with UV lamp $(275 \mathrm{~W})$ for $3 \mathrm{~h}$ at $23{ }^{\circ} \mathrm{C}$. Saturated aqueous $\mathrm{Na}_{2} \mathrm{~S}_{2} \mathrm{O}_{3}$ was added, and the two layers were separated. The organic layer was dried over $\mathrm{MgSO}_{4}$ and concentrated to give $10.7 \mathrm{~g}$ of the pure trans isomer. $\mathrm{R}_{f} 0.26\left(15 \% \mathrm{Et}_{2} \mathrm{O} /\right.$ pentane $) ;[\alpha]_{\mathrm{D}}{ }^{25}=$ 
-25.3 (c 0.25, $\left.\mathrm{CHCl}_{3}\right) ;{ }^{1} \mathrm{H} \mathrm{NMR}\left(400 \mathrm{MHz}, \mathrm{CDCl}_{3}\right) \delta 7.04(\mathrm{~s}, 1 \mathrm{H}), 6.81(\mathrm{qd}, J=16,7 \mathrm{~Hz}, 1 \mathrm{H}), 6.11$ $(\mathrm{dd}, J=16,2 \mathrm{~Hz}, 1 \mathrm{H}), 4.97(\mathrm{t}, J=6 \mathrm{~Hz}, 1 \mathrm{H}), 3.69-3.60(\mathrm{~m}, 2 \mathrm{H}), 1.85(\mathrm{~d}, J=7 \mathrm{~Hz}, 3 \mathrm{H}), 1.91-1.78(\mathrm{~m}$, $1 \mathrm{H}), 1.77-1.59(\mathrm{~m}, 3 \mathrm{H}), 0.88(\mathrm{~s}, 9 \mathrm{H}), 0.04(\mathrm{~s}, 6 \mathrm{H}) ;{ }^{13} \mathrm{C} \mathrm{NMR}\left(100 \mathrm{MHz}, \mathrm{CDCl}_{3}\right) \delta$ 172.0, 145.7, 133.5, 129.6, 119.7, 80.5, 62.3, 30.3, 28.1, 25.9, 18.9, 18.2, -5.4; IR (neat) 2953, 2856, 1753, 1253, 1099, $1079,834 \mathrm{~cm}^{-1}$; HMRS (ESI) calc. for $\mathrm{C}_{16} \mathrm{H}_{28} \mathrm{O}_{3} \mathrm{SiNa}[\mathrm{M}+\mathrm{Na}]^{+}:$319.16999; found: 319.16926.
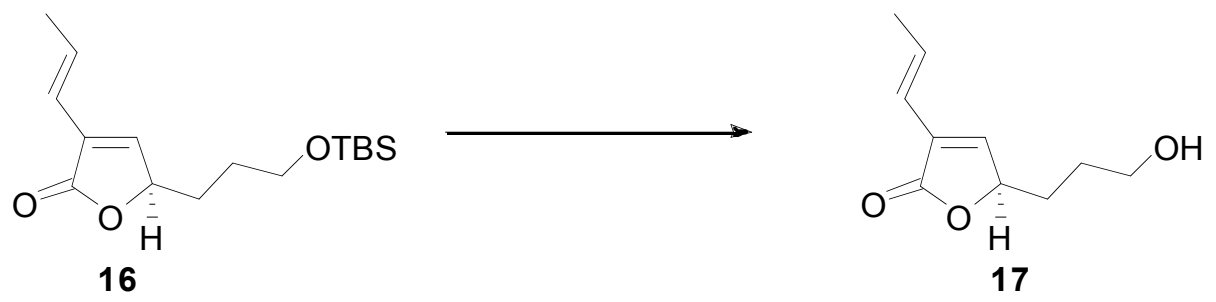

$(\boldsymbol{R})$-(E)-5-(3-Hydroxypropyl)-3-(prop-1-enyl)furan-2(5H)-one (17). To a stirred solution of silyl ether $16(3.72 \mathrm{~g}, 12.6 \mathrm{mmol})$ in dry $\mathrm{MeOH}(50 \mathrm{~mL})$ was added $\mathrm{AcCl}(90.0 \mu \mathrm{L}, 1.26 \mathrm{mmol})$ drop wise at $0{ }^{\circ} \mathrm{C}$. The reaction mixture was stirred at $23{ }^{\circ} \mathrm{C}$. After completion of the reaction (monitored by TLC), the reaction mixture was neutralized with saturated aqueous $\mathrm{NaHCO}_{3}(10 \mathrm{~mL})$ and washed with $\operatorname{AcOEt}(3 \times 50 \mathrm{~mL})$. The combined organic layers were washed with brine $(10 \mathrm{~mL})$, dried over $\mathrm{MgSO}_{4}$ and concentrated in vacuo to give $17(1.78 \mathrm{~g}, 78 \%)$ as a colorless oil after flash chromatography (50\% AcOEt/hexanes). $\mathrm{R}_{f} 0.24$ (40\% AcOEt/hexanes); $[\alpha]_{\mathrm{D}}{ }^{25}=-27.7$ (c 0.34, $\mathrm{CHCl}_{3}$ ); ${ }^{1} \mathrm{H} \mathrm{NMR}(400 \mathrm{MHz}$, $\left.\mathrm{CDCl}_{3}\right) \delta 7.03(\mathrm{~s}, 1 \mathrm{H}), 6.81(\mathrm{qd}, J=16,7 \mathrm{~Hz}, 1 \mathrm{H}), 6.12(\mathrm{dd}, J=16,2 \mathrm{~Hz}, 1 \mathrm{H}), 4.99(\mathrm{t}, J=5,5 \mathrm{~Hz}$, $1 \mathrm{H}), 3.75-3.66(\mathrm{~m}, 2 \mathrm{H}), 1.85(\mathrm{~d}, J=7 \mathrm{~Hz}, 3 \mathrm{H}), 1.95-1.81(\mathrm{~m}, 1 \mathrm{H}), 1.76-1.66(\mathrm{~m}, 3 \mathrm{H}), 1.54(\mathrm{~s}(\mathrm{br}), 1 \mathrm{H})$; ${ }^{13} \mathrm{C}$ NMR $\left(100 \mathrm{MHz}, \mathrm{CDCl}_{3}\right.$ ) $\delta 171.7,145.2,133.4,129.4,119.3,80.1,61.7,29.7,27.7,18.6$; IR (neat) 3385, 2934, 2876, 1739, 1445, 1347, 1207, $1076 \mathrm{~cm}^{-1}$; HMRS (ESI) calc. for $\mathrm{C}_{10} \mathrm{H}_{13} \mathrm{O}_{3}[\mathrm{M}-\mathrm{H}]^{+}$: 181.08690; found: 181.08692 .

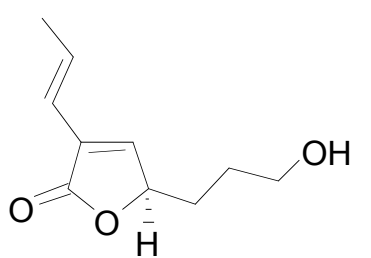

17

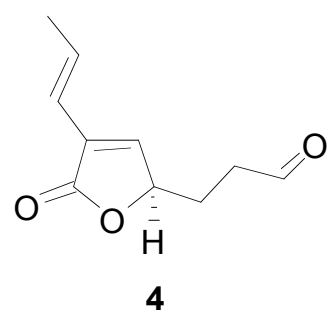

\section{IBX Oxidation:}

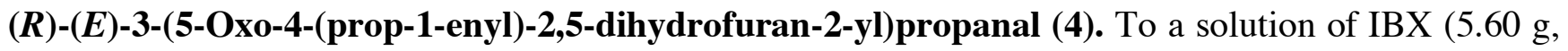
$20.0 \mathrm{mmol})$ in DMSO $(100 \mathrm{~mL})$ was added alcohol $17(1.82 \mathrm{~g}, 10 \mathrm{mmol})$. The resulting mixture was stirred $16 \mathrm{~h}$ at $23{ }^{\circ} \mathrm{C}$. The reaction was diluted with water $(70 \mathrm{~mL})$ and the white precipitate was filtrated. The filtrate was washed with $\mathrm{Et}_{2} \mathrm{O}(3 \mathrm{x} 70 \mathrm{~mL})$. The combined organic layers were washed with aqueous saturated $\mathrm{NaHCO}_{3}(30 \mathrm{~mL}), \mathrm{H}_{2} \mathrm{O}(2 \times 30 \mathrm{~mL})$ and brine $(30 \mathrm{~mL})$, dried over $\mathrm{MgSO}_{4}$ and concentrated in vacuo to give the desired product $4(1.44 \mathrm{~g}, 80 \%)$ as a colorless oil after flash chromatography (40\% AcOEt/hexanes). $\mathrm{R}_{f} 0.36$ (50\% AcOEt/hexanes); $[\alpha]_{\mathrm{D}}{ }^{25}=-42.3\left(\mathrm{c} 1.04, \mathrm{CHCl}_{3}\right)$; ${ }^{1} \mathrm{H}$ NMR $\left(300 \mathrm{MHz}, \mathrm{CDCl}_{3}\right) \delta 9.80(\mathrm{~s}, 1 \mathrm{H}), 6.99(\mathrm{~s}, 1 \mathrm{H}), 6.82(\mathrm{qd}, J=16,7 \mathrm{~Hz}, 1 \mathrm{H}), 6.10(\mathrm{dd}, J=16$, $2 \mathrm{~Hz}, 1 \mathrm{H}), 5.07-4.94(\mathrm{~m}, 1 \mathrm{H}), 2.78-2.52(\mathrm{~m}, 2 \mathrm{H}), 2.33-2.10(\mathrm{~m}, 1 \mathrm{H}), 1.85$ (d, J = $7 \mathrm{~Hz}, 3 \mathrm{H}), 1.90-1.74$ $(\mathrm{m}, 1 \mathrm{H}) ;{ }^{13} \mathrm{C}$ NMR $\left(100 \mathrm{MHz}, \mathrm{CDCl}_{3}\right) \delta 200.4,171.5,144.7,134.0,129.9,119.4,79.0,38.7,25.4$, 18.8; IR (neat) 2916, 2729, 1748, 1723, 1444, 1347, $1075 \mathrm{~cm}^{-1}$; HMRS (ESI) calc. for $\mathrm{C}_{10} \mathrm{H}_{13} \mathrm{O}_{3}$ $[\mathrm{M}+\mathrm{H}]^{+}:$181.08592; found: 181.08534 . 


\section{Swern Oxidation:}

(R)-(E)-3-(5-Oxo-4-(prop-1-enyl)-2,5-dihydrofuran-2-yl)propanal (4). A solution of primary alcohol 17 (91 mg, $0.5 \mathrm{mmol})$ in $\mathrm{CH}_{2} \mathrm{Cl}_{2}(3 \mathrm{~mL})$ was mixed with Swern reagent prepared from $(\mathrm{COCl})_{2}(0.087$ $\mathrm{mL}, 1 \mathrm{mmol})$ and DMSO $(0.14 \mathrm{~mL}, 2 \mathrm{mmol})$ in $\mathrm{CH}_{2} \mathrm{Cl}_{2}(3 \mathrm{~mL})$ at $-78{ }^{\circ} \mathrm{C}$. After 20 min stirring, $\mathrm{Et}_{3} \mathrm{~N}$ $(0.42 \mathrm{~mL}, 3 \mathrm{mmol})$ was added to the mixture at $-78^{\circ} \mathrm{C}$ and the stirring was continued for additional 30 min at $0{ }^{\circ} \mathrm{C}$. The reaction was quenched by the addition of water $(5 \mathrm{~mL})$. The two layers were separated and the aqueous layer was washed with $\mathrm{CH}_{2} \mathrm{Cl}_{2}(3 \times 10 \mathrm{~mL})$. The combined organic layers were dried over $\mathrm{MgSO}_{4}$ and concentrated to give the desired product 4 (65 mg, 72\%) as colorless oil after flash chromatography (40\% AcOEt/hexanes). The NMR spectra were identical to those obtained for 4 using IBX oxidation.

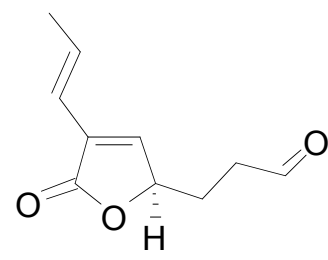

4

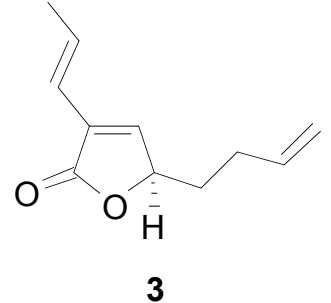

3

(-)-Pregaliellalactone (3). To a solution of (IMes) $\mathrm{CuCl}(40 \mathrm{mg}, 0.10 \mathrm{mmol})$ and triphenylphosphine $(288 \mathrm{mg}, 1.10 \mathrm{mmol})$ in dioxane $(15 \mathrm{~mL})$ at $23{ }^{\circ} \mathrm{C}$. was added 2-propanol $(0.76 \mathrm{~mL}, 10 \mathrm{mmol})$ followed by the aldehyde $4(180 \mathrm{mg}, 1.00 \mathrm{mmol})$. The reaction mixture was heated at $60{ }^{\circ} \mathrm{C}$ and trimethylsilyldiazomethane $(1.4 \mathrm{mmol})$ was then added drop wise. The reaction was stirred for $2 \mathrm{~h}$. Water $(10 \mathrm{~mL})$ was added and the two layers were separated. The aqueous layer was washed with pentane $(6 \times 30 \mathrm{~mL})$. The combined organic layers were washed with water $(3 \times 10 \mathrm{~mL})$ and brine $(2 \times$ $10 \mathrm{~mL}$ ), dried over $\mathrm{MgSO}_{4}$ and concentrated in vacuo to give the desired product $3(137 \mathrm{mg}, 77 \%)$ as a colorless oil after flash chromatography $\left(10 \% \mathrm{Et}_{2} \mathrm{O} /\right.$ pentane $) . \mathrm{R}_{f} 0.43(20 \% \mathrm{AcOEt} / \mathrm{hexanes}) ;[\alpha]_{\mathrm{D}}{ }^{25}=$ -38.0 (c 1.4, $\left.\mathrm{CHCl}_{3}\right) ;{ }^{1} \mathrm{H}$ NMR $\left(400 \mathrm{MHz}, \mathrm{CDCl}_{3}\right) \delta 7.02(\mathrm{~s}, 1 \mathrm{H}), 6.87-6.75(\mathrm{~m}, 1 \mathrm{H}), 6.12(\mathrm{~d}, J=15 \mathrm{~Hz}$, $1 \mathrm{H}), 5.86-5.74(\mathrm{~m}, 1 \mathrm{H}), 5.11-5.00(\mathrm{~m}, 2 \mathrm{H}), 4.98-4.92(\mathrm{~m}, 1 \mathrm{H}), 2.29-2.17(\mathrm{~m}, 2 \mathrm{H}), 1.85(\mathrm{~d}, J=7 \mathrm{~Hz}$, 3H), 1.82-1.67 (m, 2H); ${ }^{13} \mathrm{C}$ NMR $\left(125 \mathrm{MHz}, \mathrm{CDCl}_{3}\right) \delta$ 172.0, 145.4, 136.8, 133.7, 129.7, 119.7, 116.0, 79.9, 32.8, 29.2, 18.9; IR (neat) 2917, 2850, 1750, 1438, 1111, $1083 \mathrm{~cm}^{-1}$; HMRS (ESI) calc. for $\mathrm{C}_{11} \mathrm{H}_{15} \mathrm{O}_{2}[\mathrm{M}+\mathrm{H}]^{+}:$176.1066; found: 179.1070 .

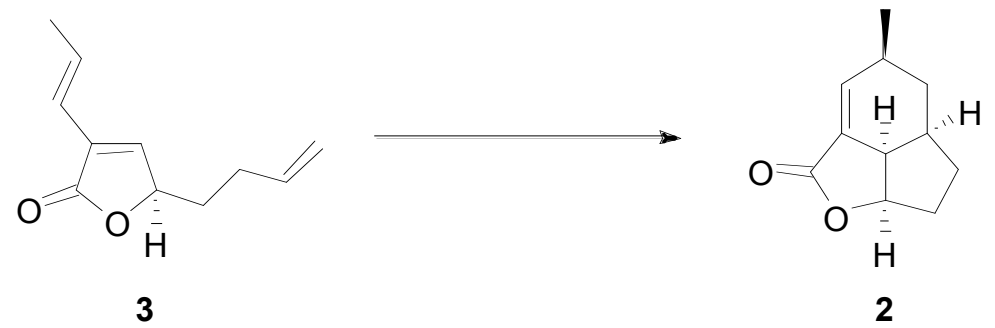

(+)-Desoxygaliellalactone (2). To a solution of pregaliellalactone 3 (89 $\mathrm{mg}, 0.50 \mathrm{mmol})$ in dioxane (10 $\mathrm{mL})$ was added $\mathrm{AlCl}_{3}(72 \mathrm{mg}, 0.55 \mathrm{mmol})$. The reaction mixture was heated at $140{ }^{\circ} \mathrm{C}$ under microwaves for $2 \mathrm{~h}$. The reaction mixture was concentrated in vacuo to give desoxygaliellalactone 2 (25 $\mathrm{mg}, 56 \%)$ after flash chromatography $\left(20 \% \mathrm{Et}_{2} \mathrm{O} /\right.$ pentane $) . \mathrm{R}_{f} 0.16\left(20 \% \mathrm{Et}_{2} \mathrm{O} /\right.$ pentane $) ;[\alpha]_{\mathrm{D}}{ }^{25}=+71.3$ (c 0.99, $\left.\mathrm{CHCl}_{3}\right) ;{ }^{1} \mathrm{H}$ NMR $\left(500 \mathrm{MHz}, \mathrm{CDCl}_{3}\right) \delta 6.85(\mathrm{t}, J=3 \mathrm{~Hz}, 1 \mathrm{H}), 5.08-5.03(\mathrm{~m}, 1 \mathrm{H}), 3.10-3.04(\mathrm{~m}$, $1 \mathrm{H}), 2.51-2.41(\mathrm{~m}, 1 \mathrm{H}), 2.21-2.09(\mathrm{~m}, 2 \mathrm{H}), 1.92-1.82(\mathrm{~m}, 2 \mathrm{H}), 1.82-1.75(\mathrm{~m}, 1 \mathrm{H}), 1.21(\mathrm{~d}, J=7 \mathrm{~Hz}$, $3 \mathrm{H}), 1.19-1.14(\mathrm{~m}, 1 \mathrm{H}), 0.85-0.78(\mathrm{~m}, 1 \mathrm{H}) ;{ }^{13} \mathrm{C} \mathrm{NMR}\left(100 \mathrm{MHz}, \mathrm{CDCl}_{3}\right) \delta$ 170.5, 144.8, 129.9, 83.5, 44.2, 37.3, 34.5, 33.7, 31.6, 31.4, 20.1; IR (neat) 2956, 2929, 2864, 1754, 1667, 1208, $1017 \mathrm{~cm}^{-1}$; HMRS (ESI) calc. for $\mathrm{C}_{11} \mathrm{H}_{15} \mathrm{O}_{2}[\mathrm{M}+\mathrm{H}]^{+}:$179.1066; found: 179.1074 . 

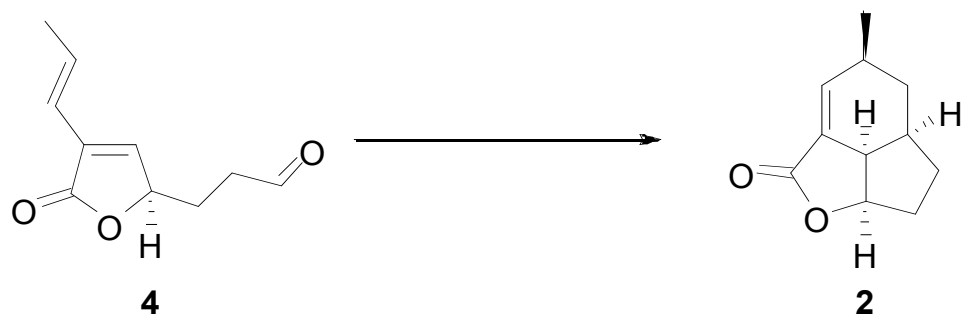

(+)-Desoxygaliellalactone (2). To a solution of (IMes) $\mathrm{CuCl}(40 \mathrm{mg}, 0.10 \mathrm{mmol})$ and triphenylphosphine $(288 \mathrm{mg}, 1.10 \mathrm{mmol})$ in dioxane $(15 \mathrm{~mL})$ at $23^{\circ} \mathrm{C}$ was added 2-propanol $(0.76 \mathrm{~mL}$, $10 \mathrm{mmol})$ followed by the aldehyde $4(180 \mathrm{mg}, 1.00 \mathrm{mmol})$. The reaction mixture was heated at $60{ }^{\circ} \mathrm{C}$ and trimethylsilyldiazomethane $(1.4 \mathrm{mmol})$ was added drop wise. The reaction was stirred for $2 \mathrm{~h}$, then $\mathrm{AlCl}_{3}(146 \mathrm{mg}, 1.10 \mathrm{mmol})$ was added to the reaction mixture and stirred $16 \mathrm{~h}$ at $140{ }^{\circ} \mathrm{C}$. The reaction mixture was concentrated in vacuo to give desoxygalliellalactone $2(92 \mathrm{mg}, 52 \%)$ after flash chromatography (20\% $\mathrm{Et}_{2} \mathrm{O} /$ pentane). $\mathrm{R}_{f} 0.16\left(20 \% \mathrm{Et}_{2} \mathrm{O} /\right.$ pentane); $[\alpha]_{\mathrm{D}}{ }^{25}=+71.3$ (c $\left.0.99, \mathrm{CHCl}_{3}\right) ;{ }^{1} \mathrm{H}$ NMR (500 MHz, $\left.\mathrm{CDCl}_{3}\right) \delta 6.85(\mathrm{t}, J=3 \mathrm{~Hz}, 1 \mathrm{H}), 5.08-5.03(\mathrm{~m}, 1 \mathrm{H}), 3.10-3.04(\mathrm{~m}, 1 \mathrm{H}), 2.51-2.41(\mathrm{~m}$, $1 \mathrm{H}), 2.21-2.09(\mathrm{~m}, 2 \mathrm{H}), 1.92-1.82(\mathrm{~m}, 2 \mathrm{H}), 1.82-1.75(\mathrm{~m}, 1 \mathrm{H}), 1.21(\mathrm{~d}, J=7 \mathrm{~Hz}, 3 \mathrm{H}), 1.19-1.14(\mathrm{~m}$, $1 \mathrm{H}), 0.85-0.78(\mathrm{~m}, 1 \mathrm{H}) ;{ }^{13} \mathrm{C}$ NMR $\left(125 \mathrm{MHz}, \mathrm{CDCl}_{3}\right) \delta 170.5,144.8,129.9,83.5,44.2,37.3,34.5$, 33.7, 31.7, 31.4, 20.1; IR (neat) 2956, 2929, 2864, 1754, 1667, 1208, $1017 \mathrm{~cm}^{-1}$; HMRS (ESI) calc. for $\mathrm{C}_{11} \mathrm{H}_{15} \mathrm{O}_{2}[\mathrm{M}+\mathrm{H}]^{+}$: 179.1066; found: 179.1074. 


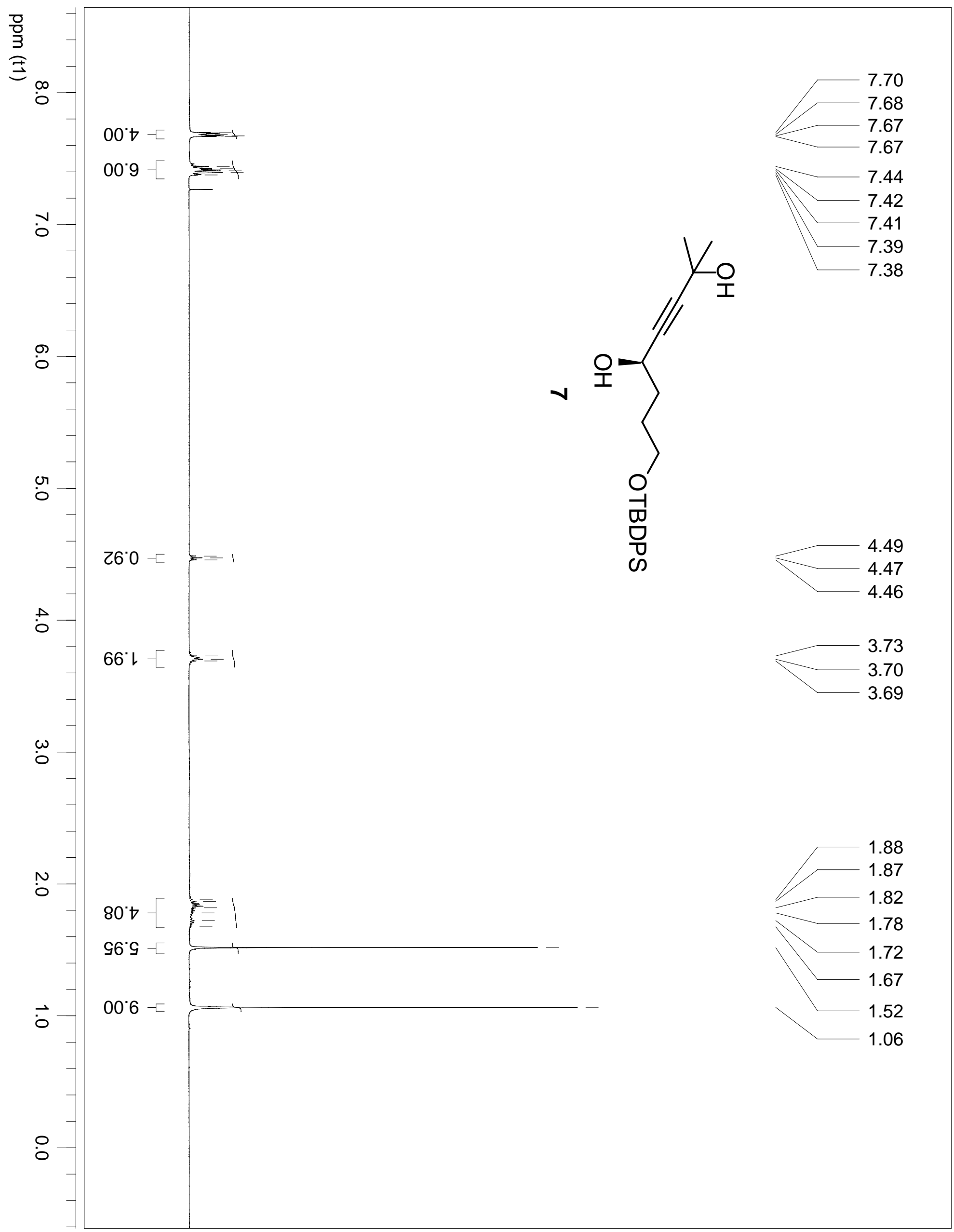




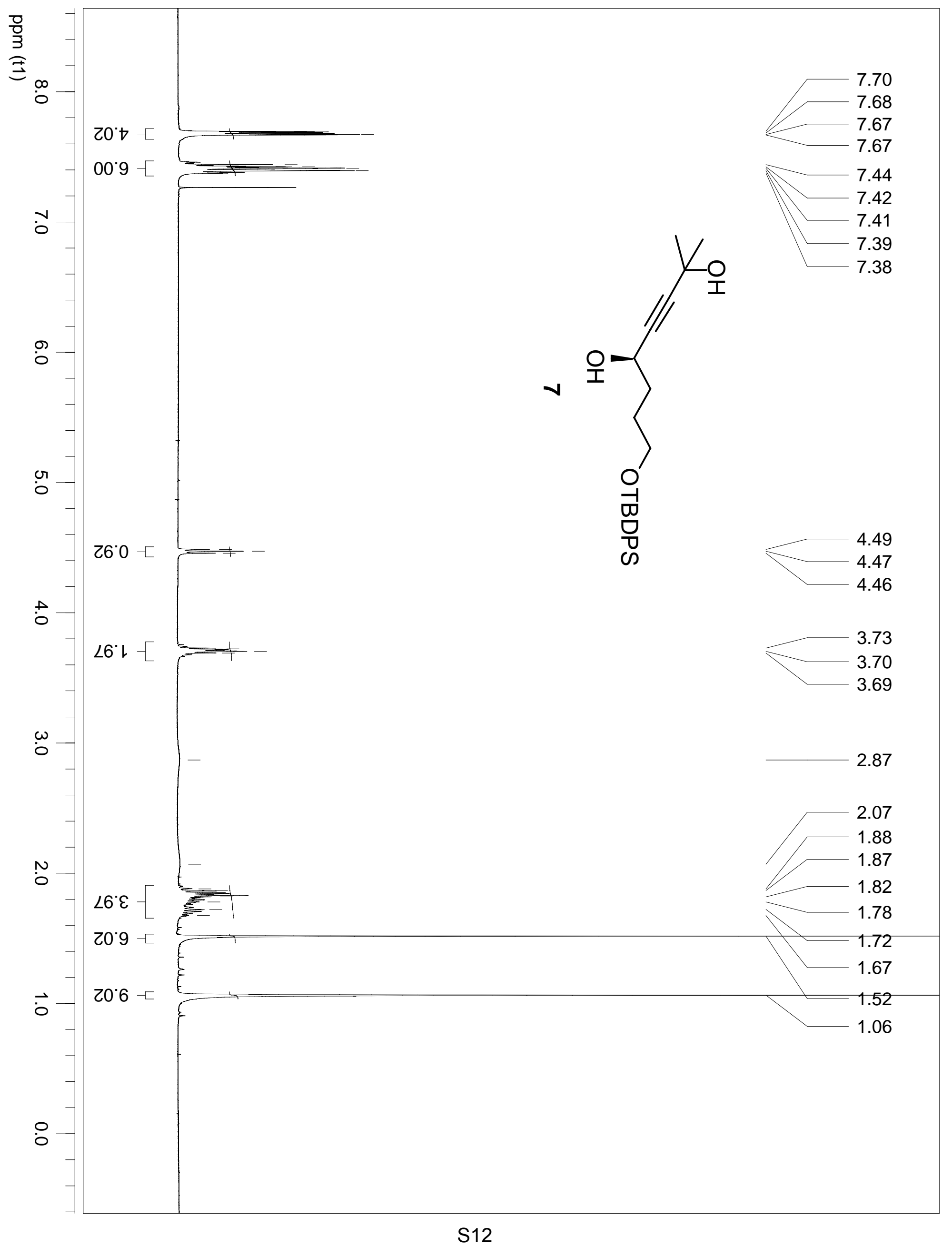




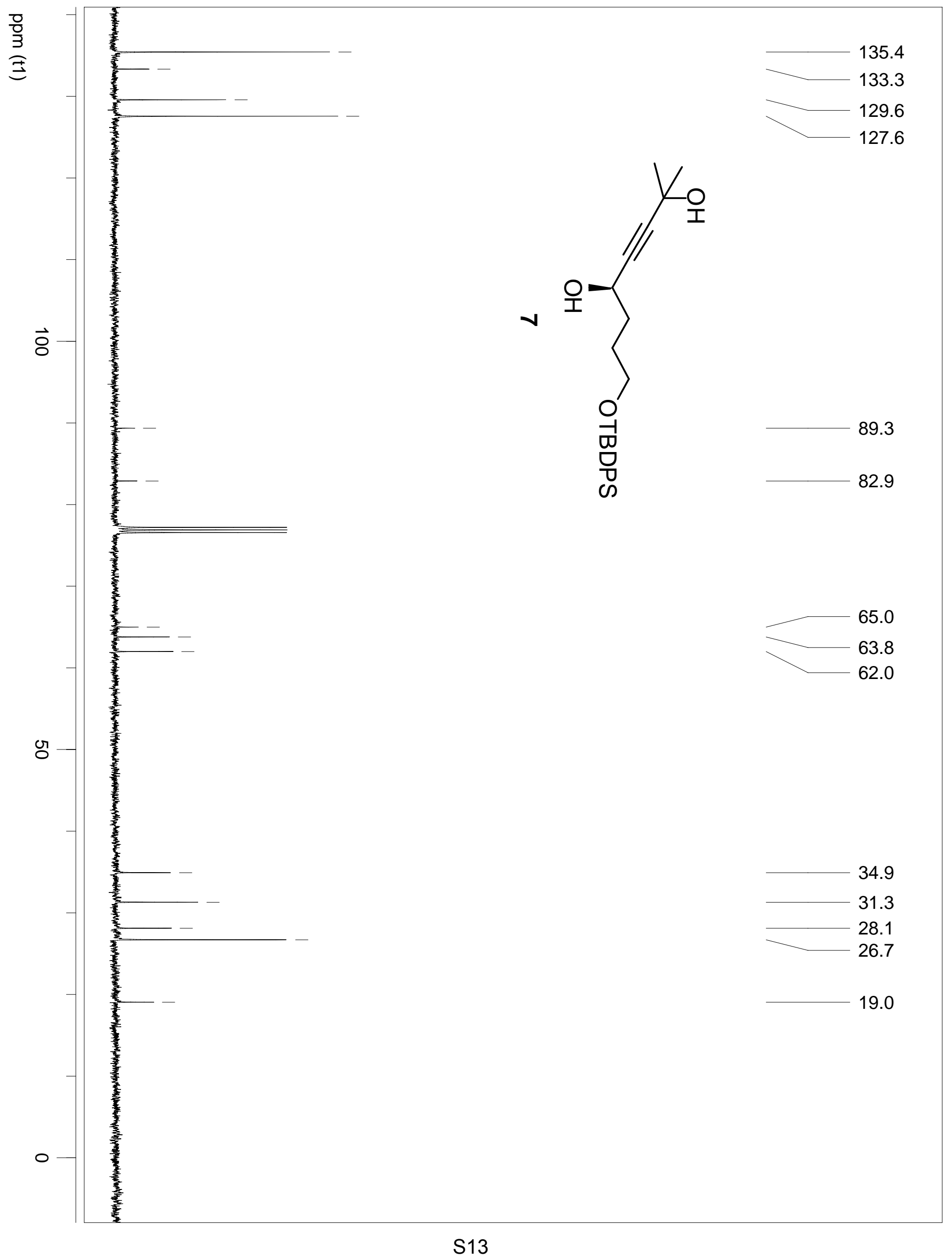




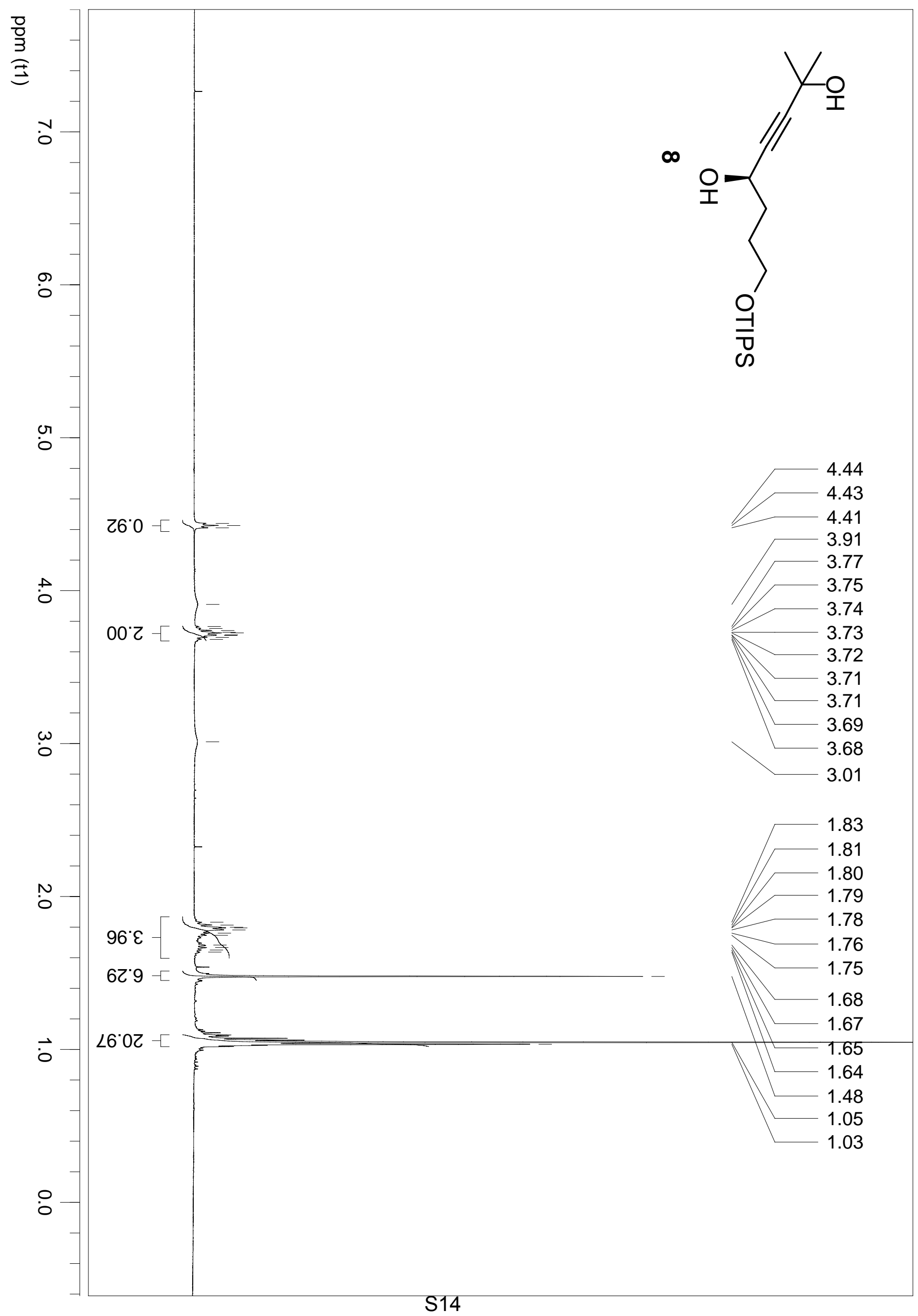




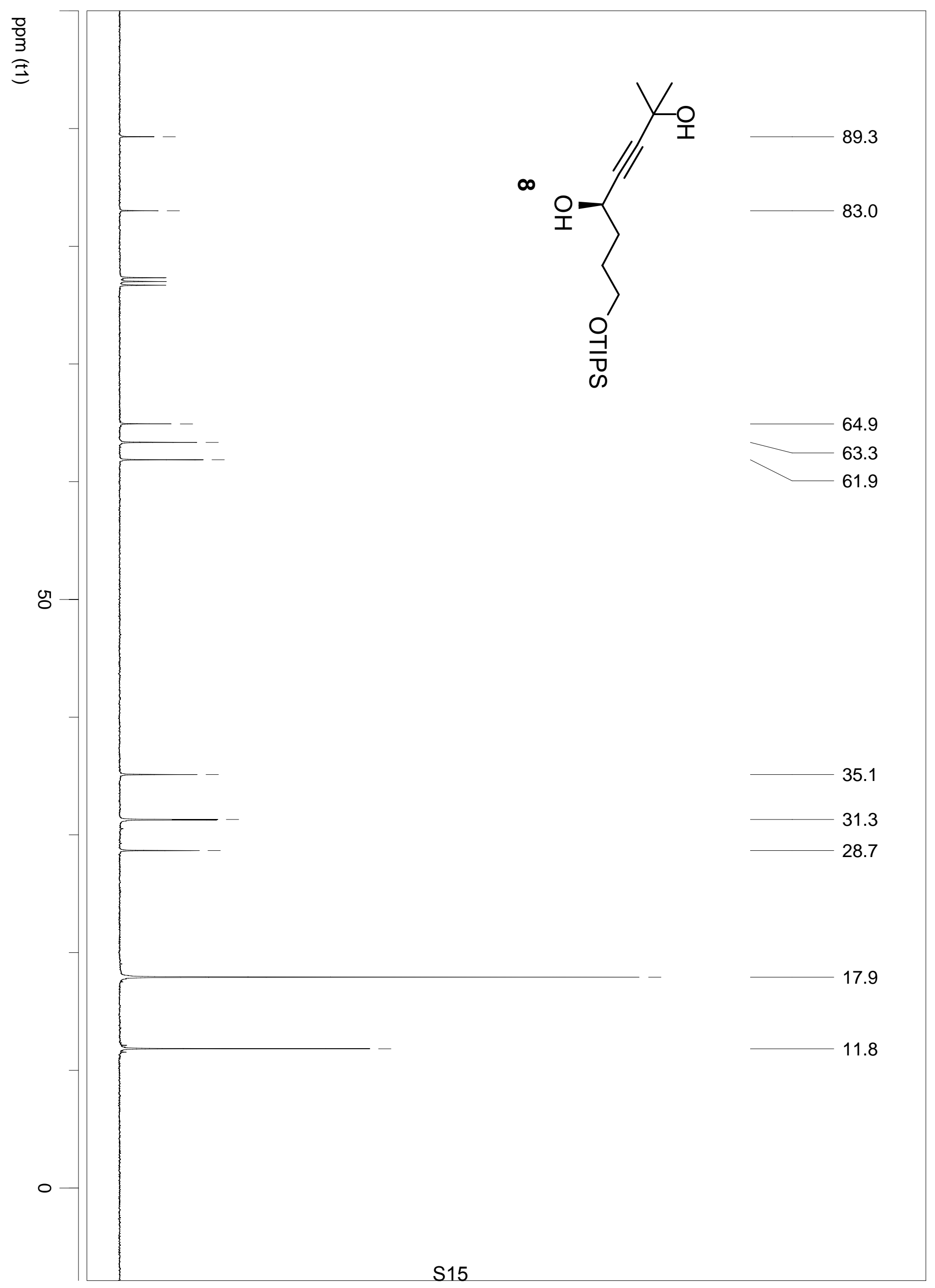




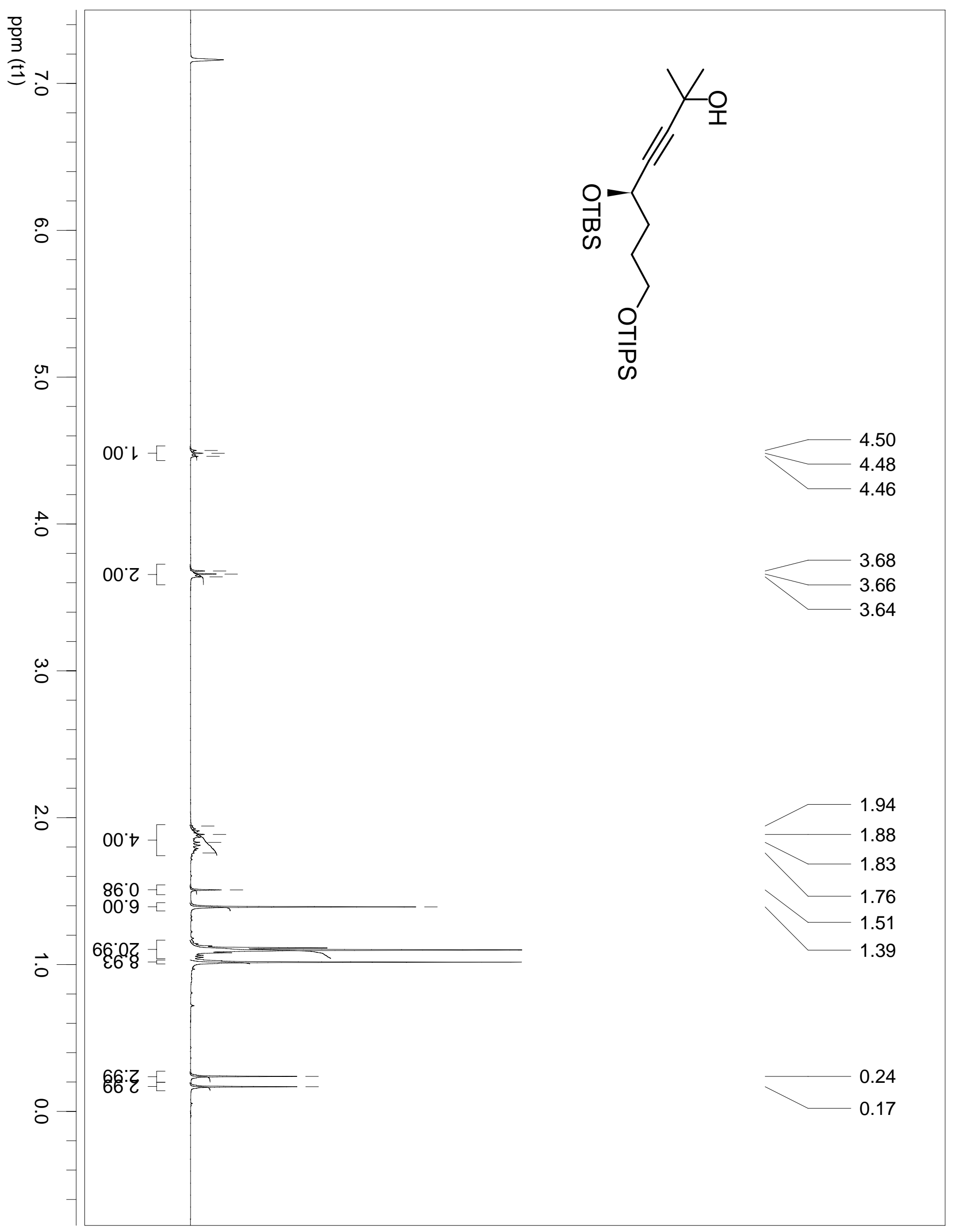




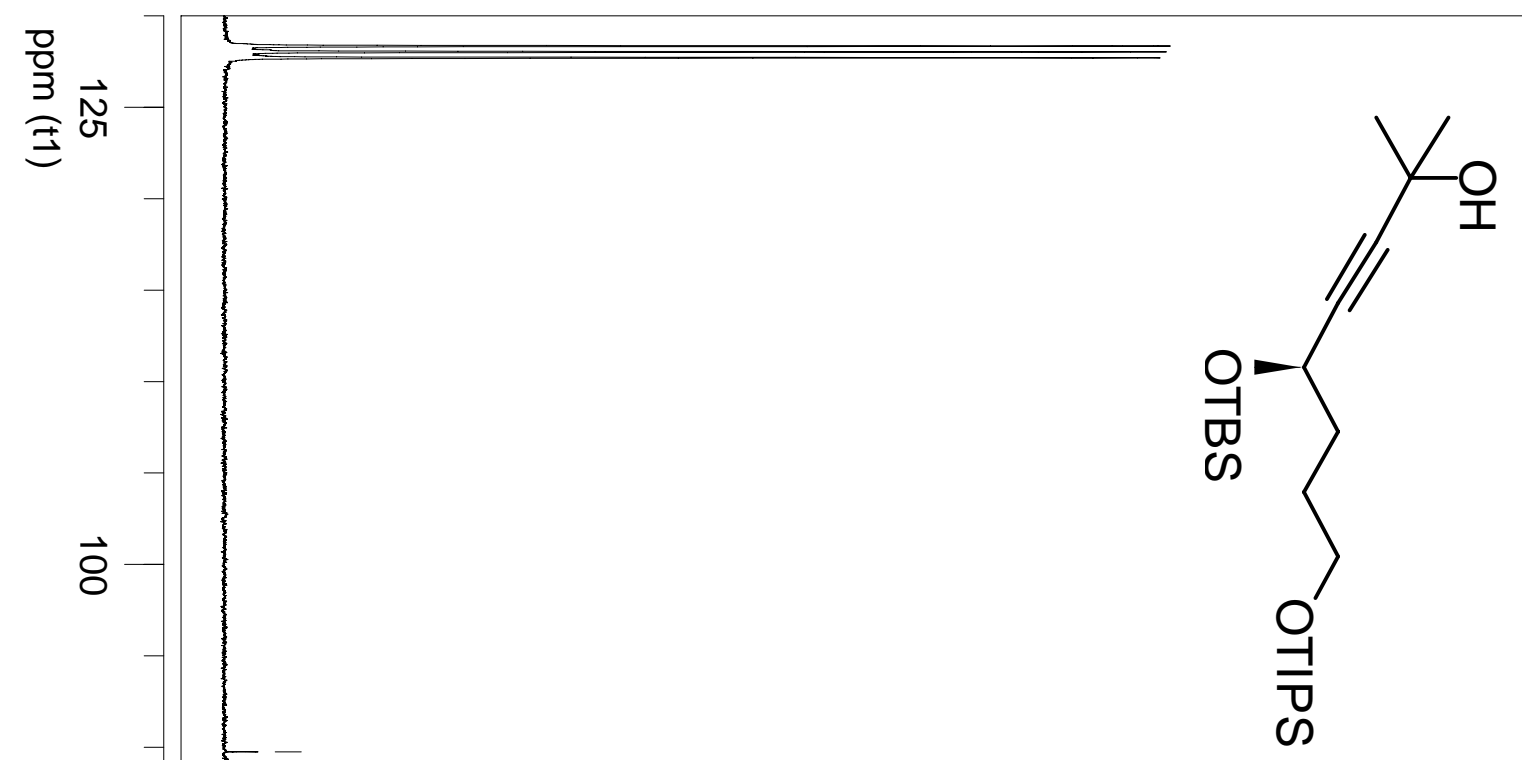

89.7

83.7

64.8

63.4

63.3

35.8

31.6

31.5

29.3

26.0

18.3

12.3

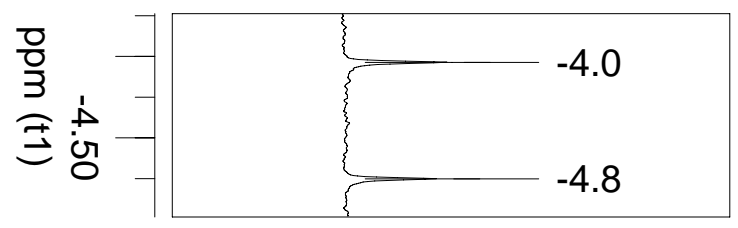

$-4.0$ 


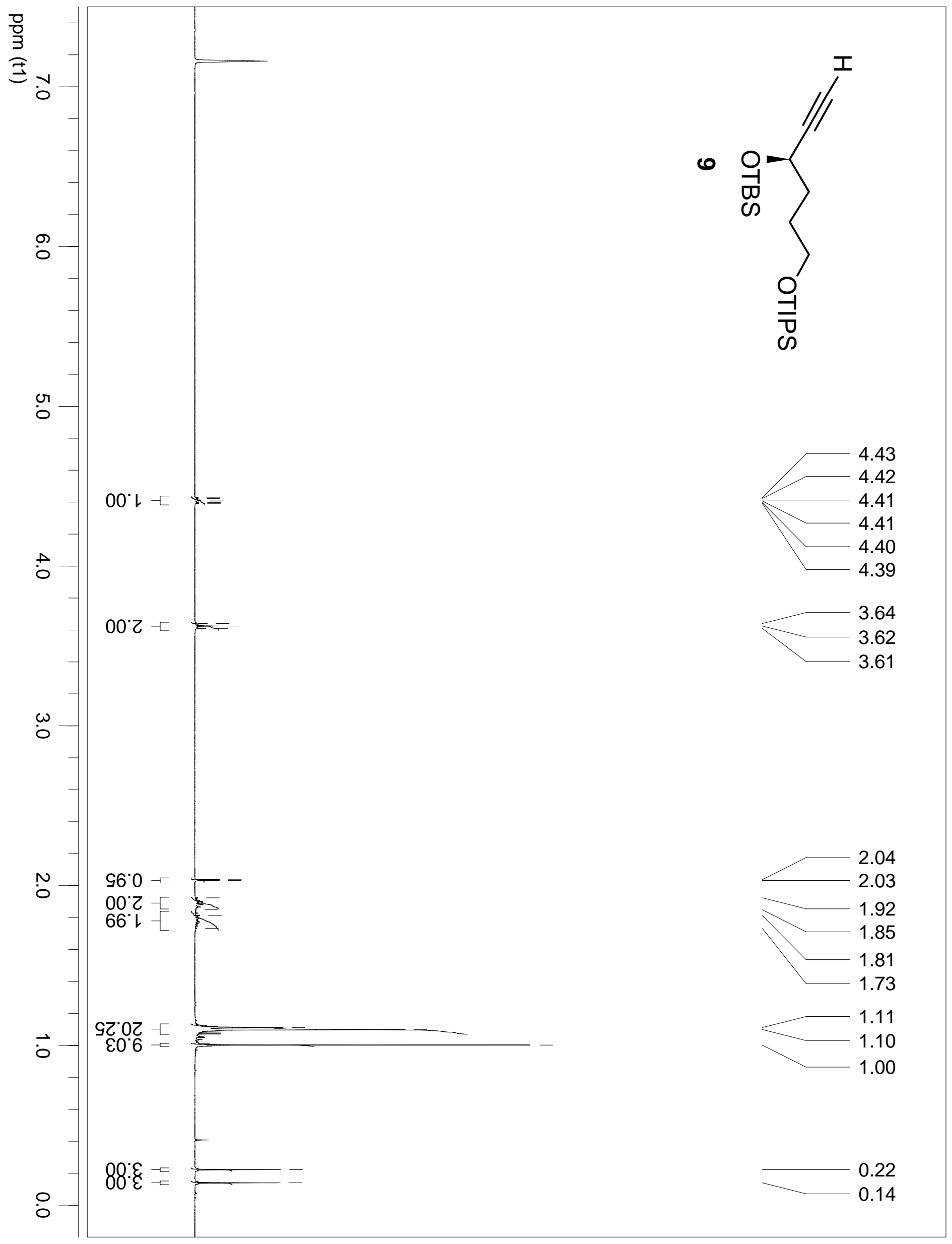




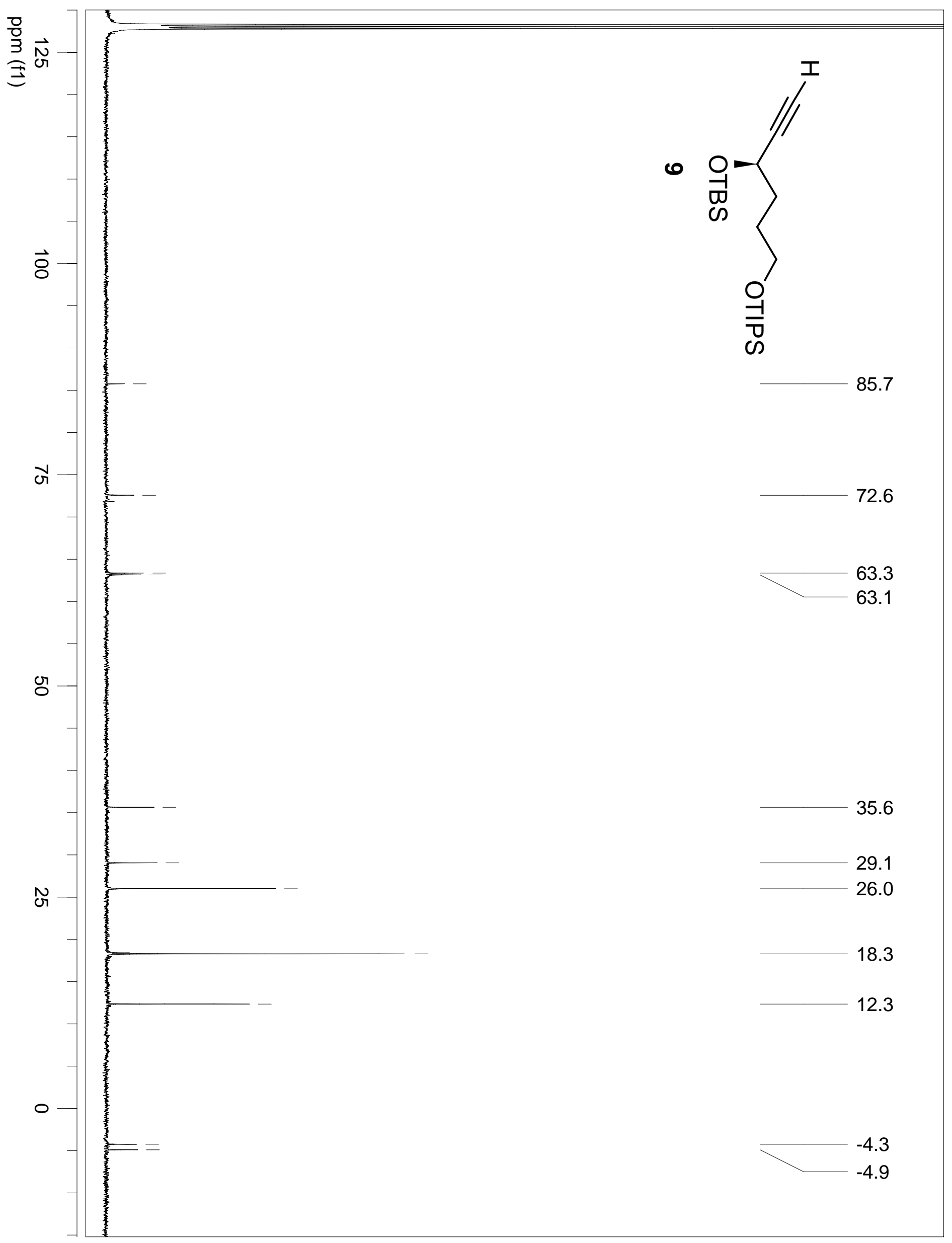




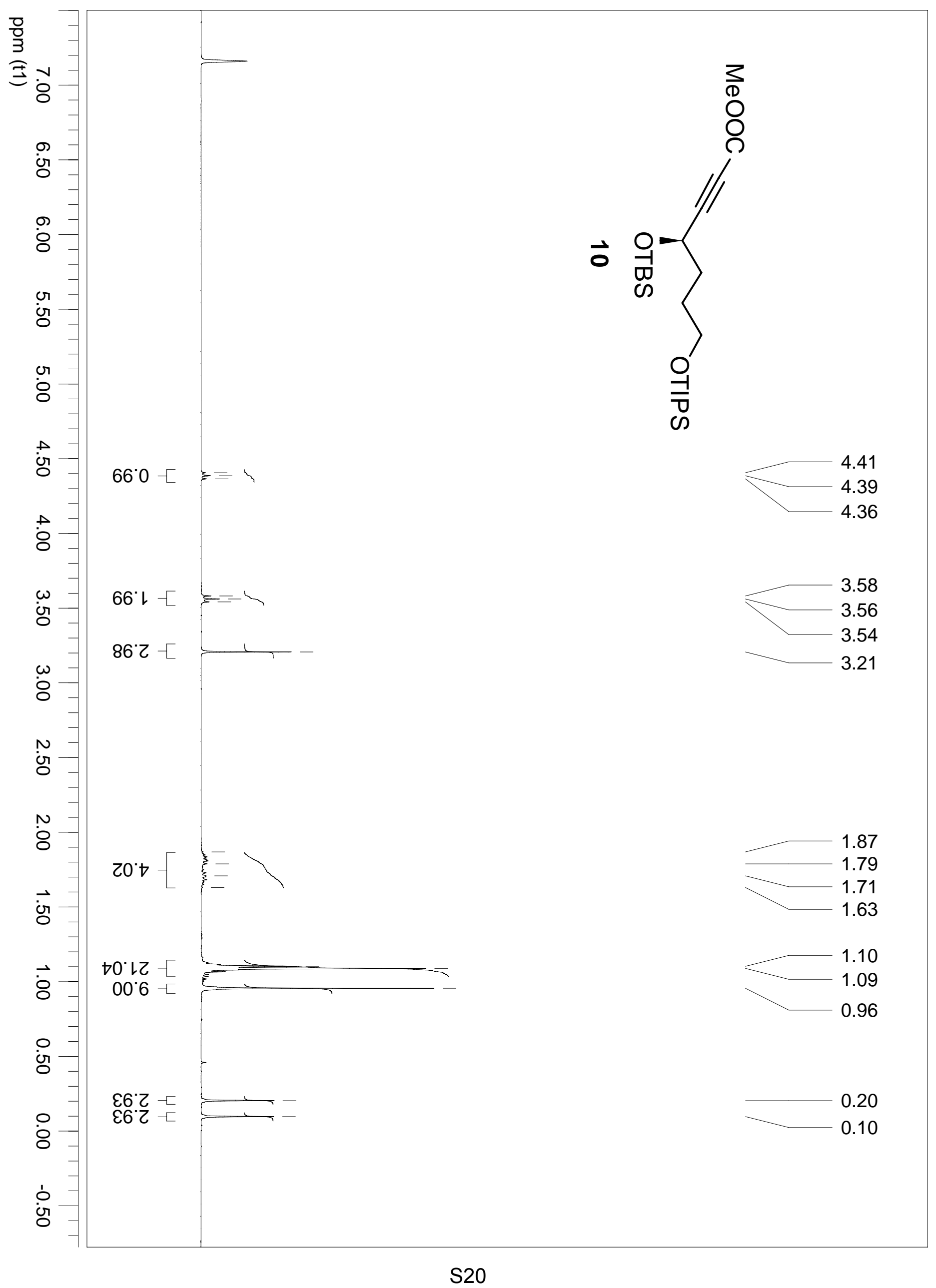




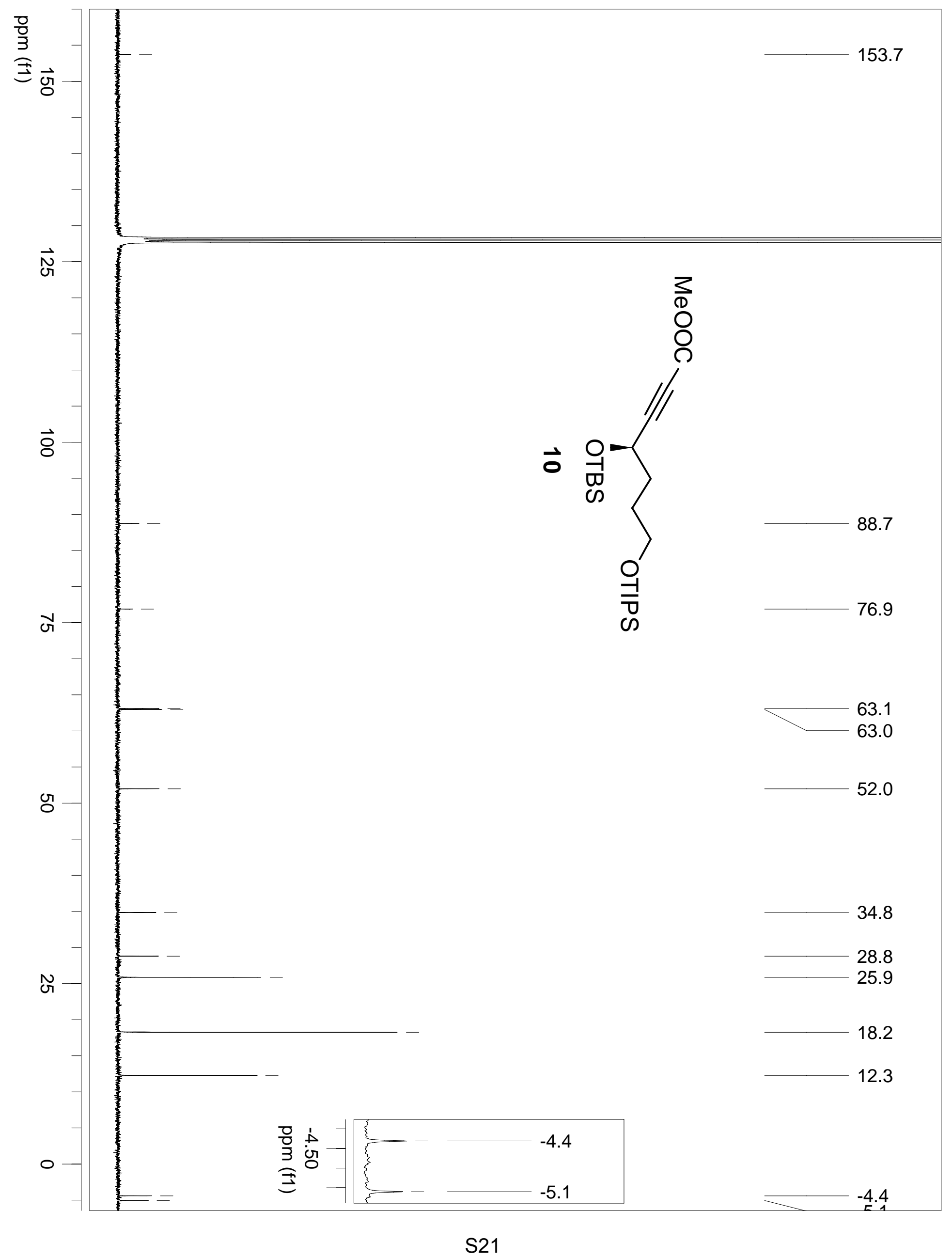




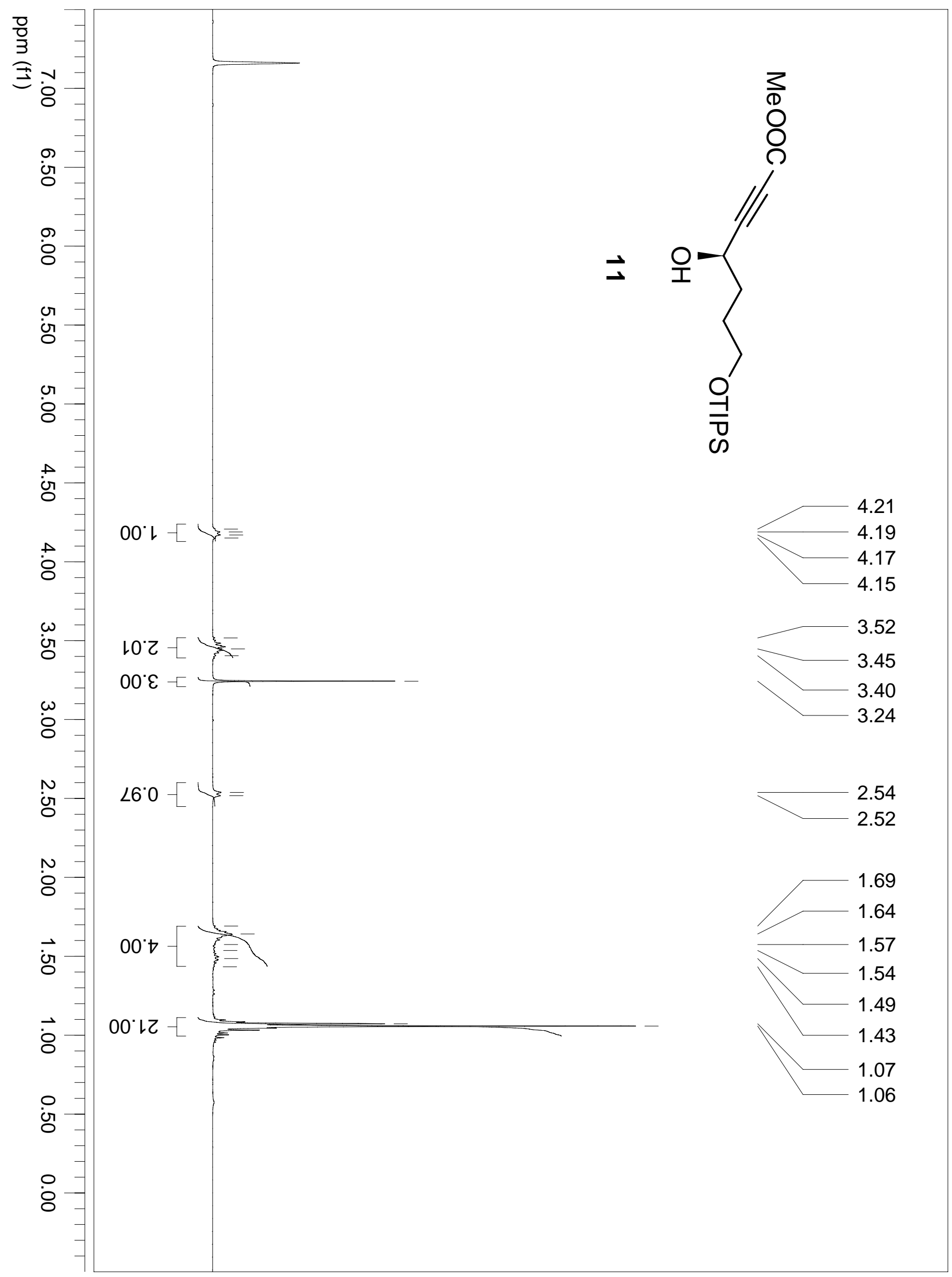




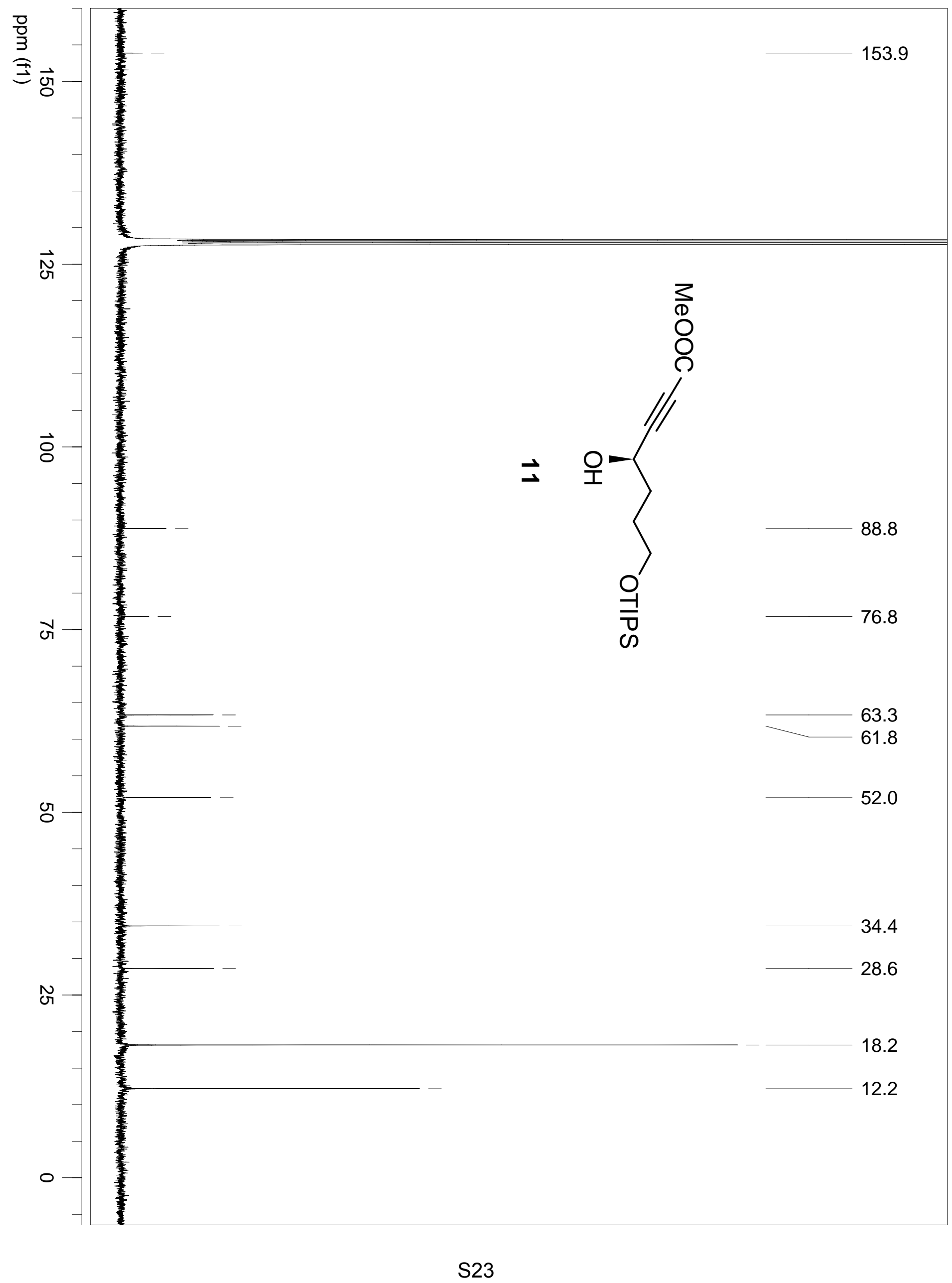




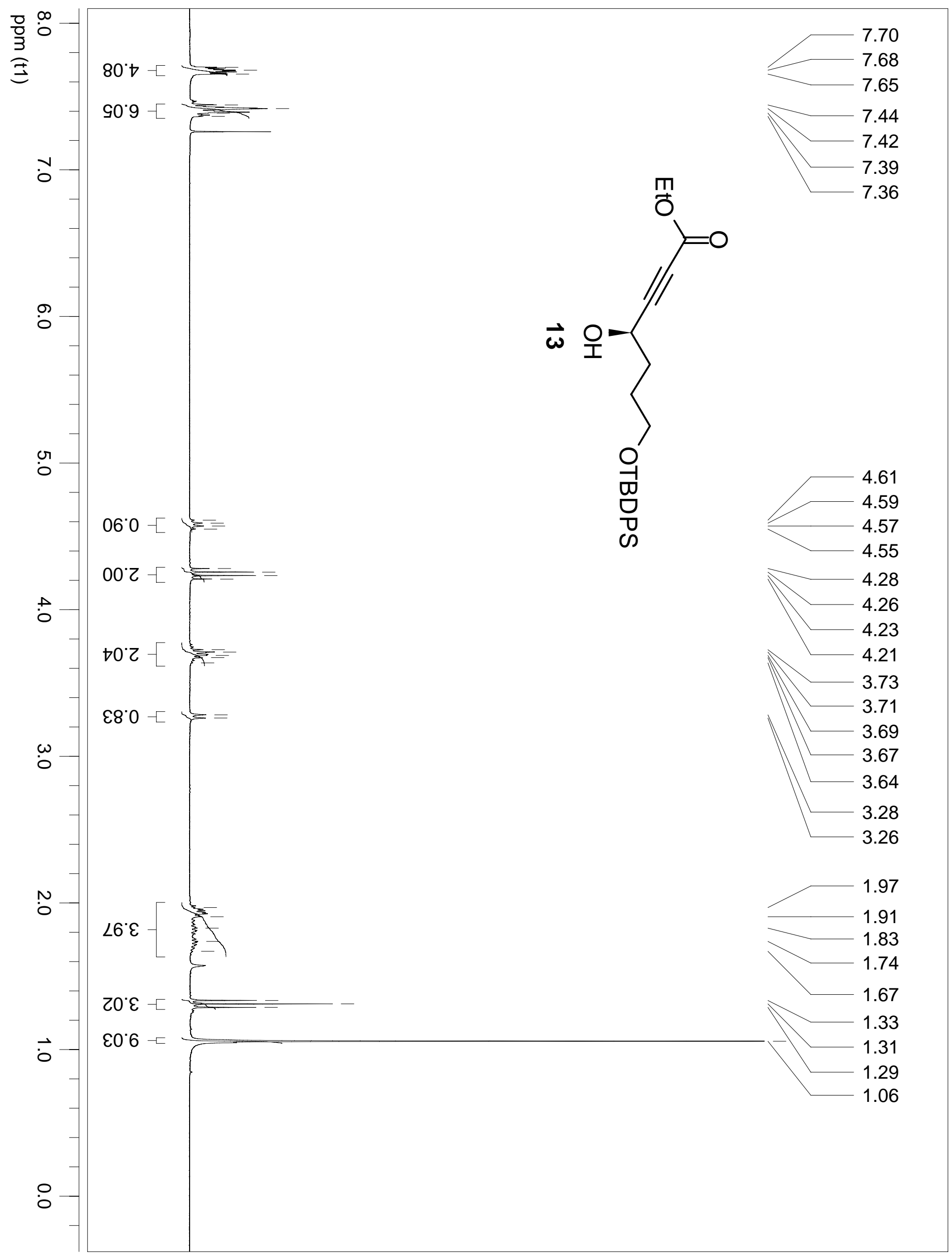




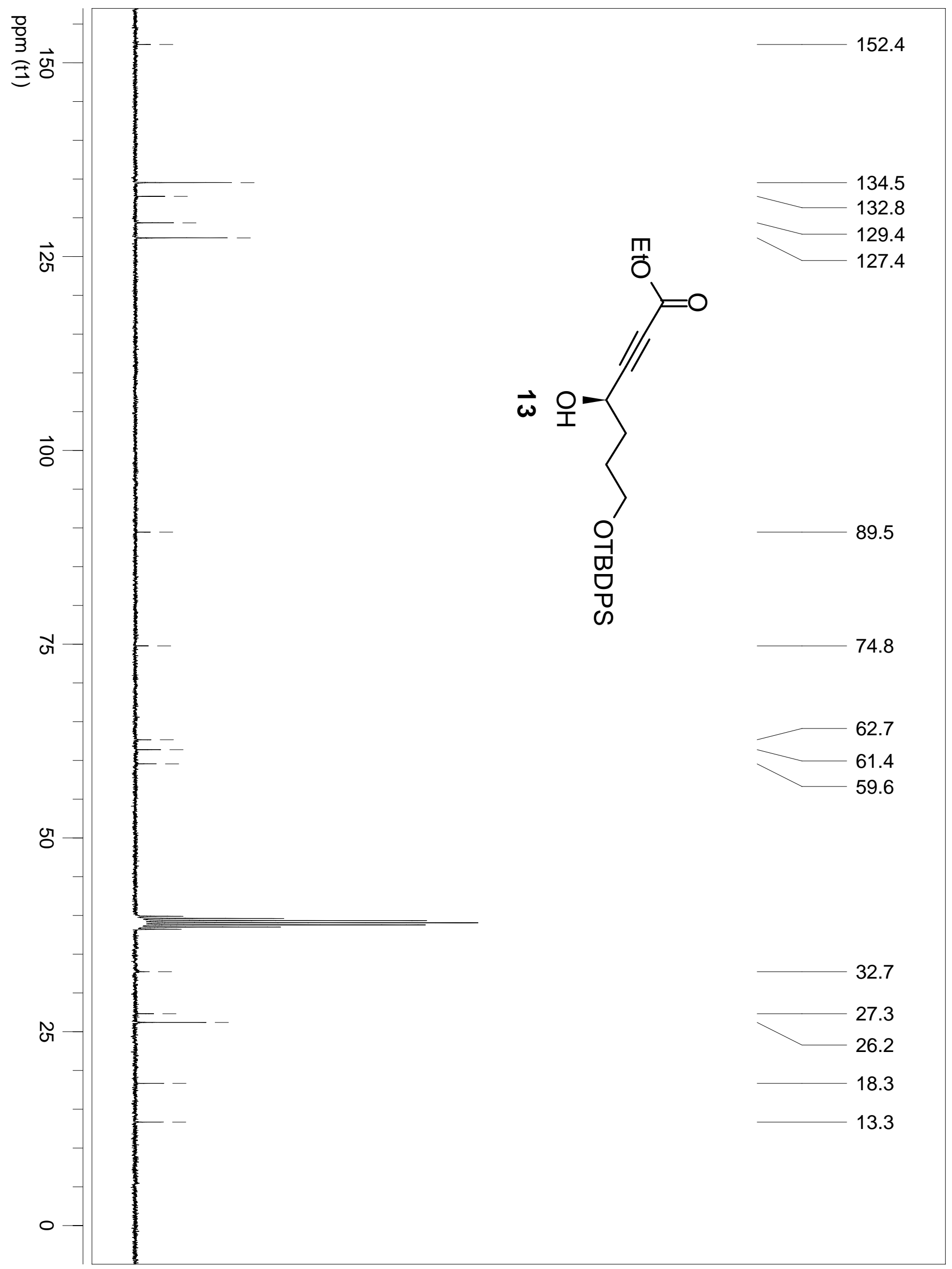




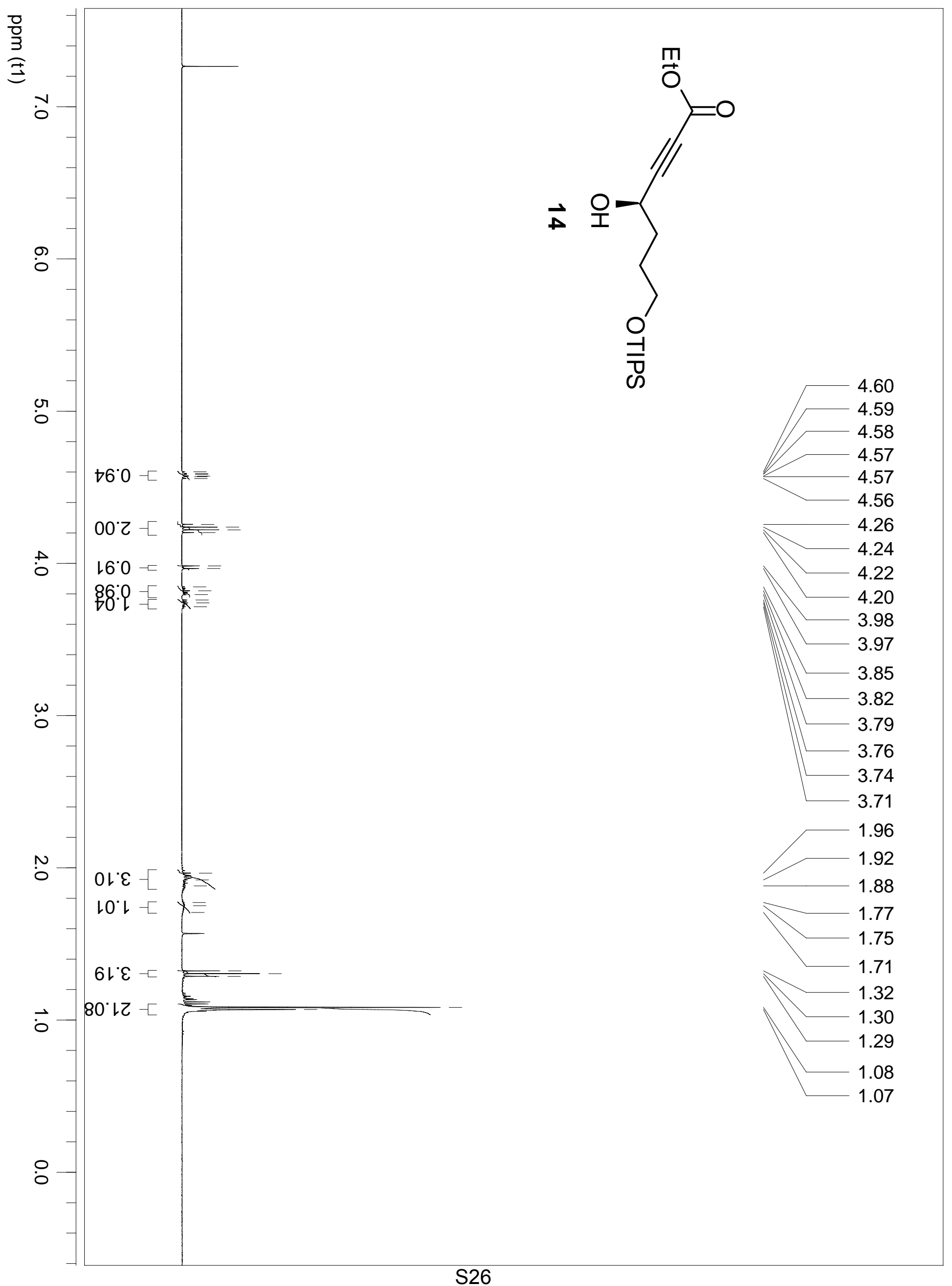




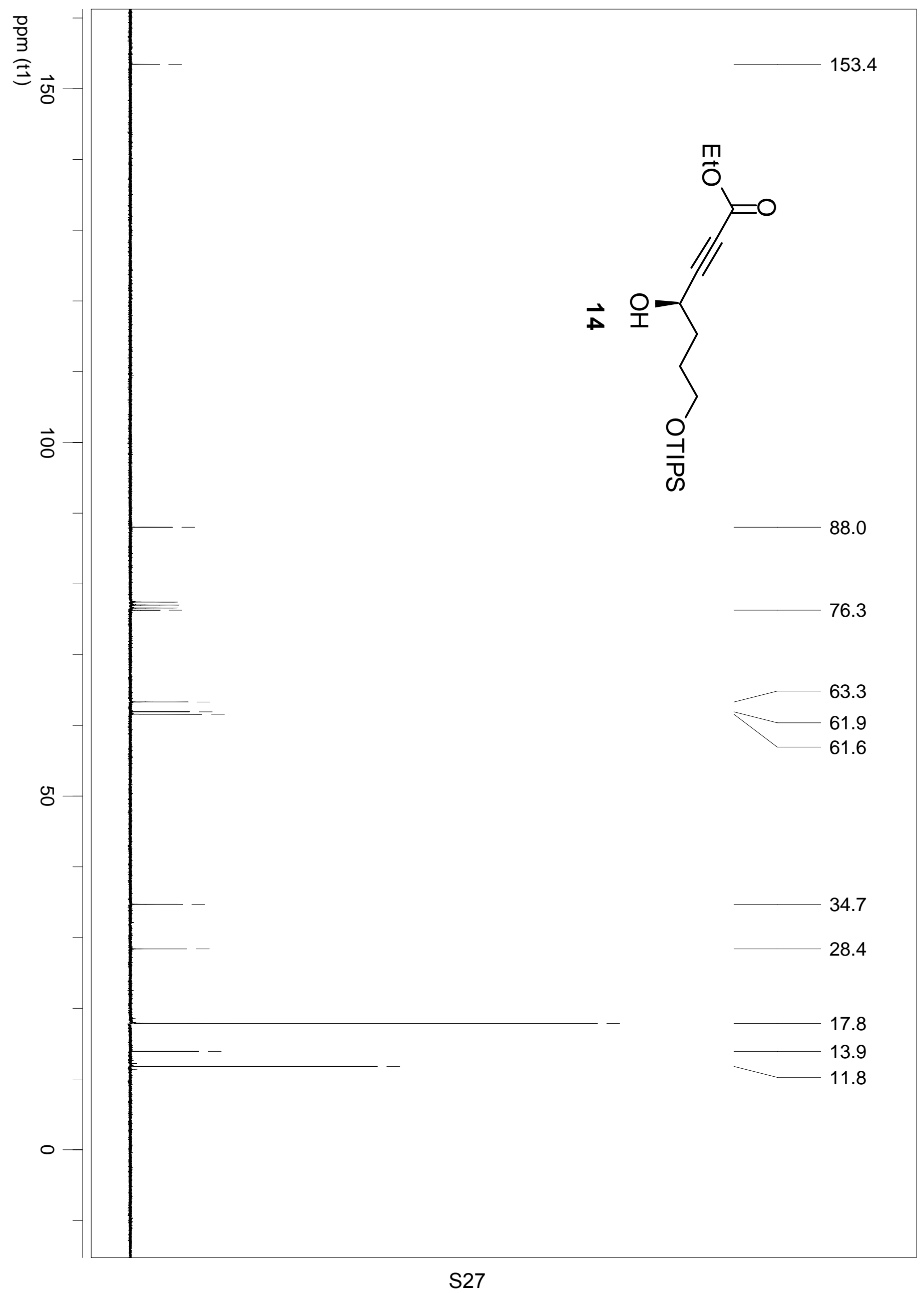




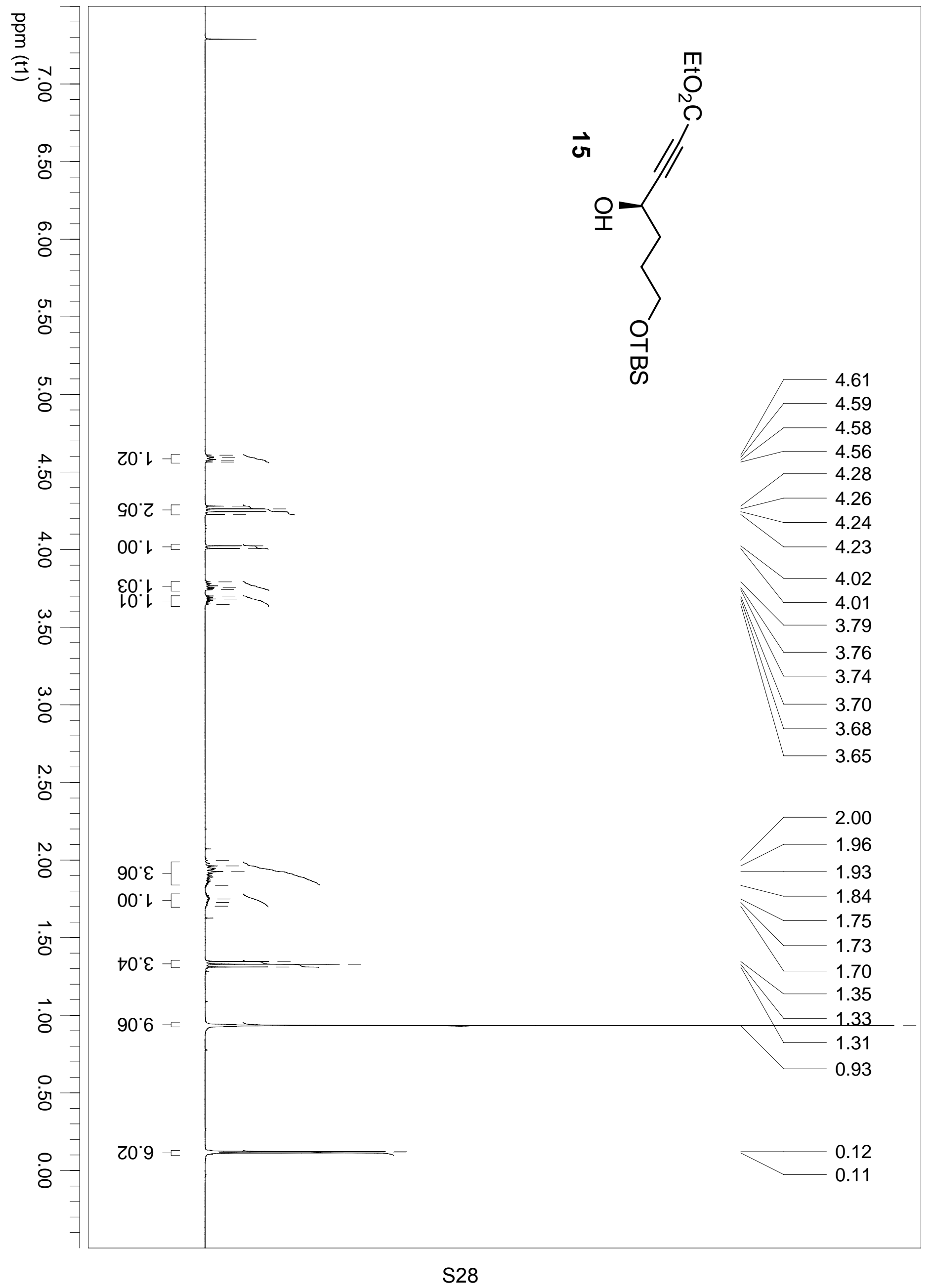




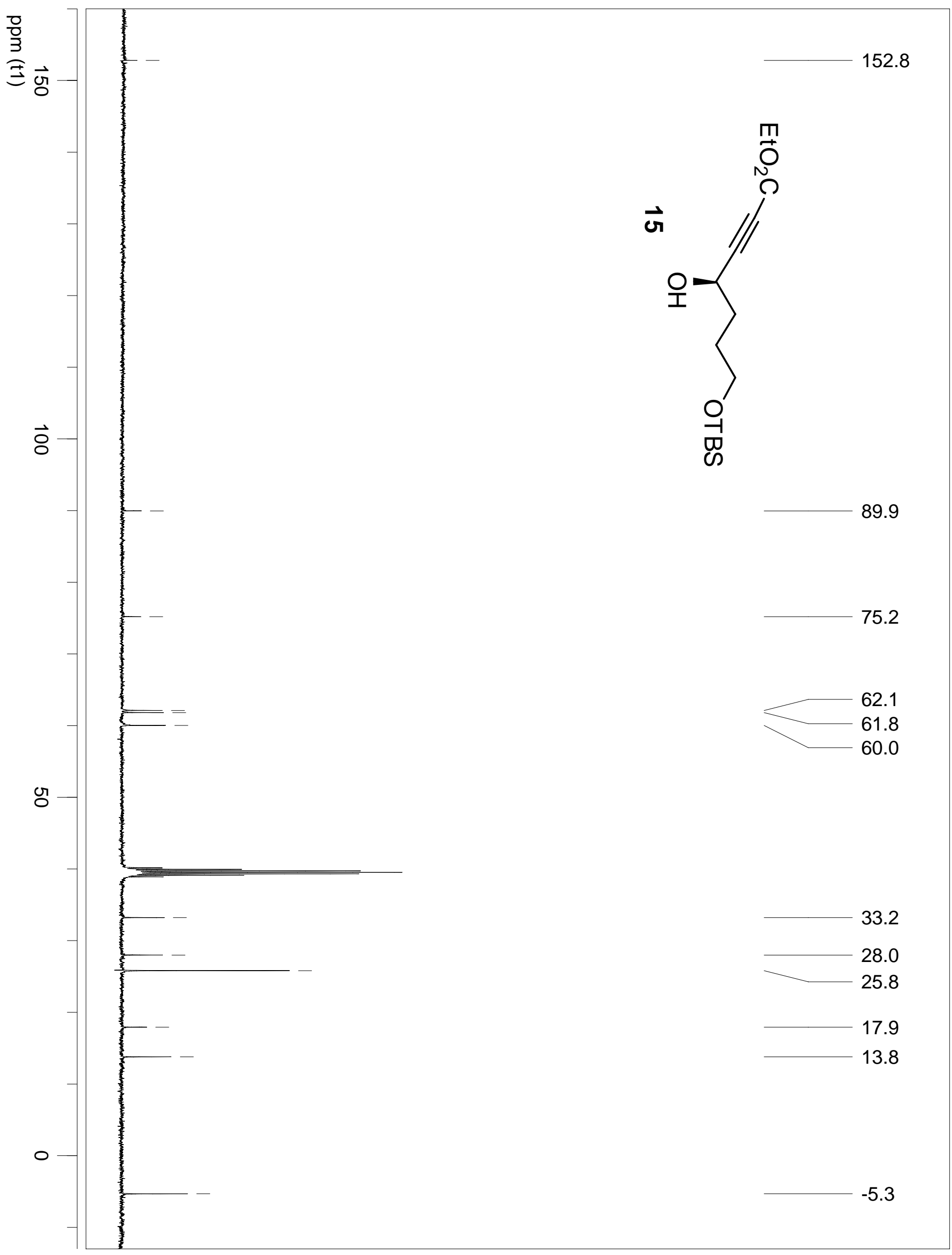




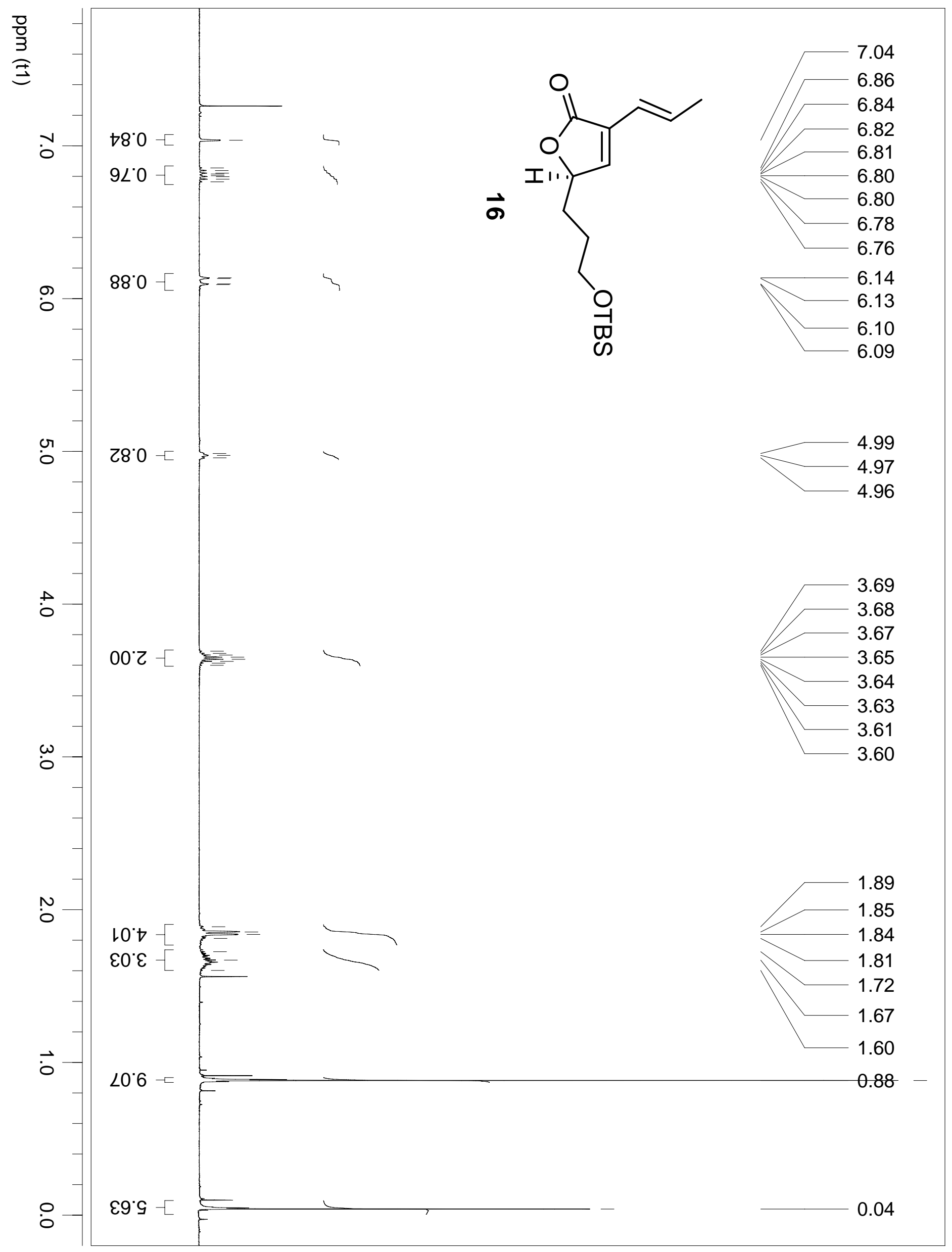




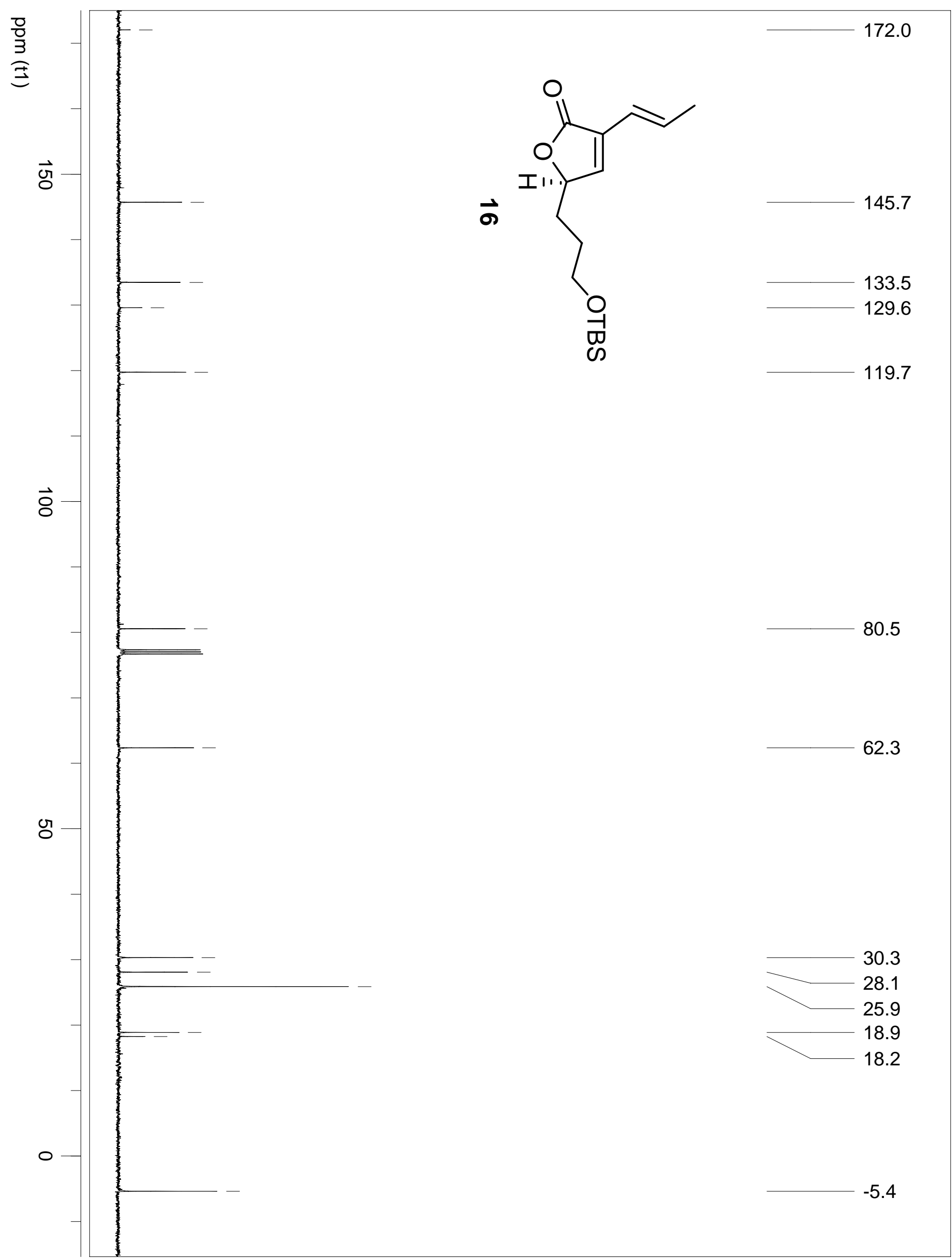




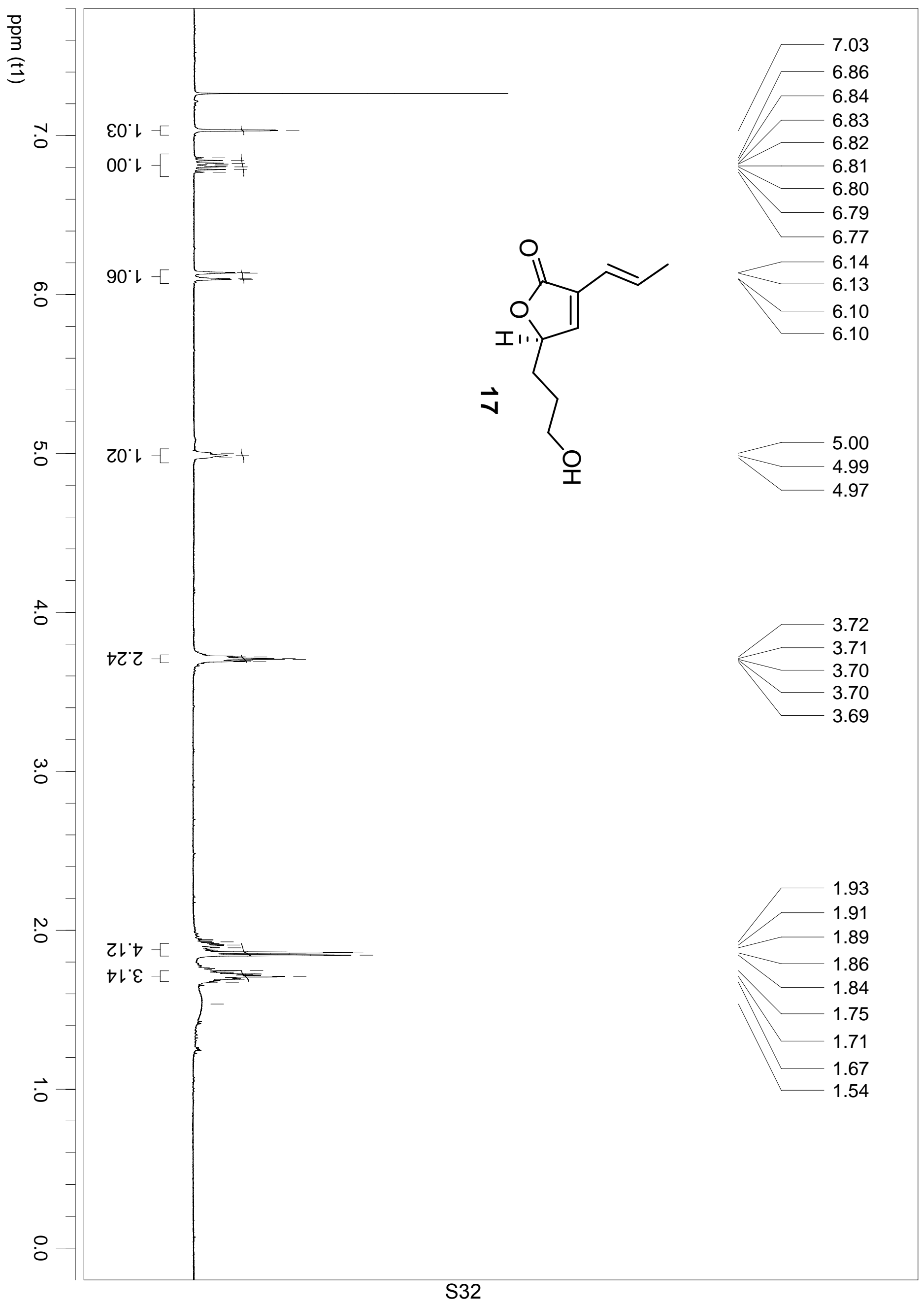




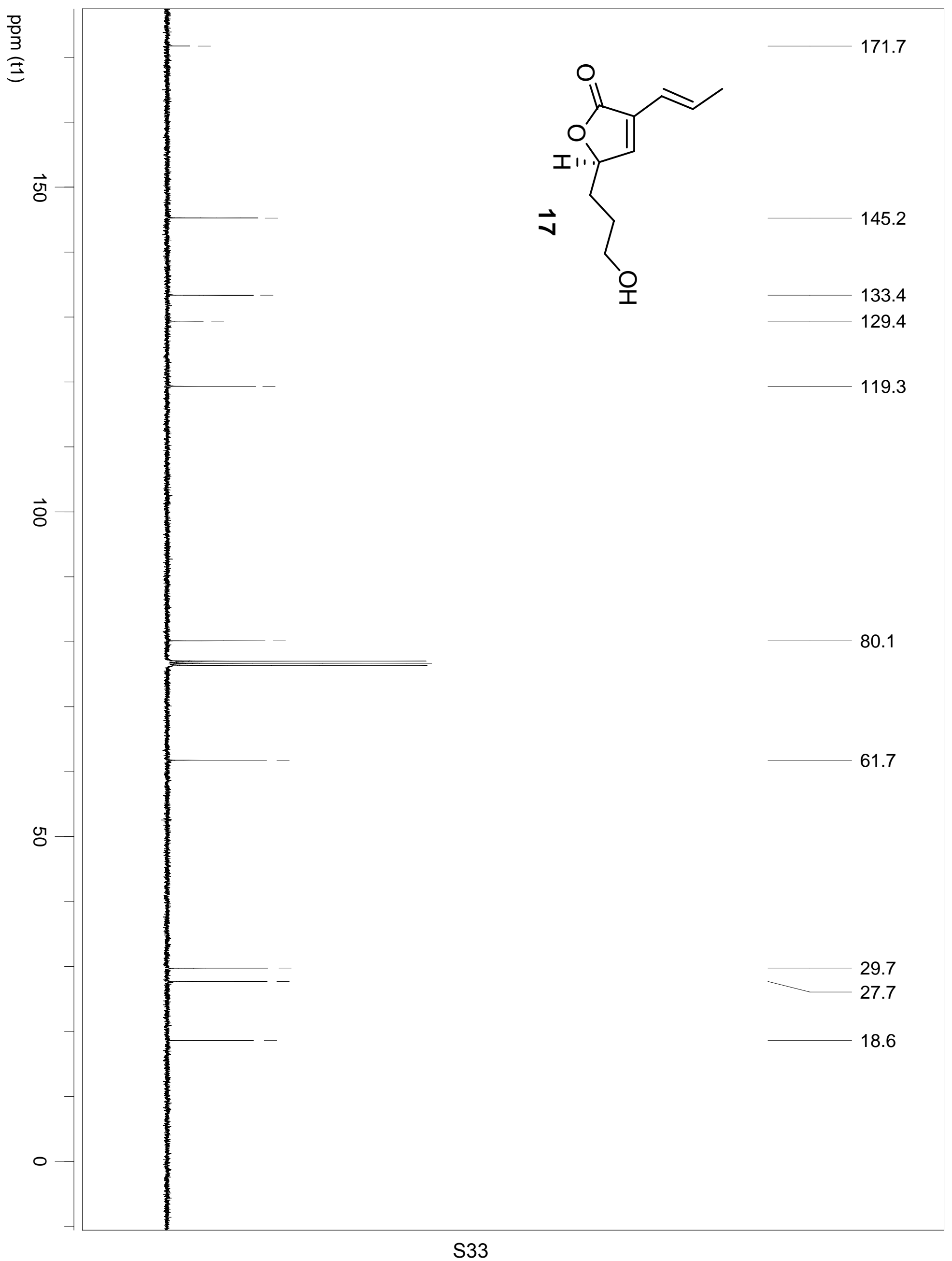




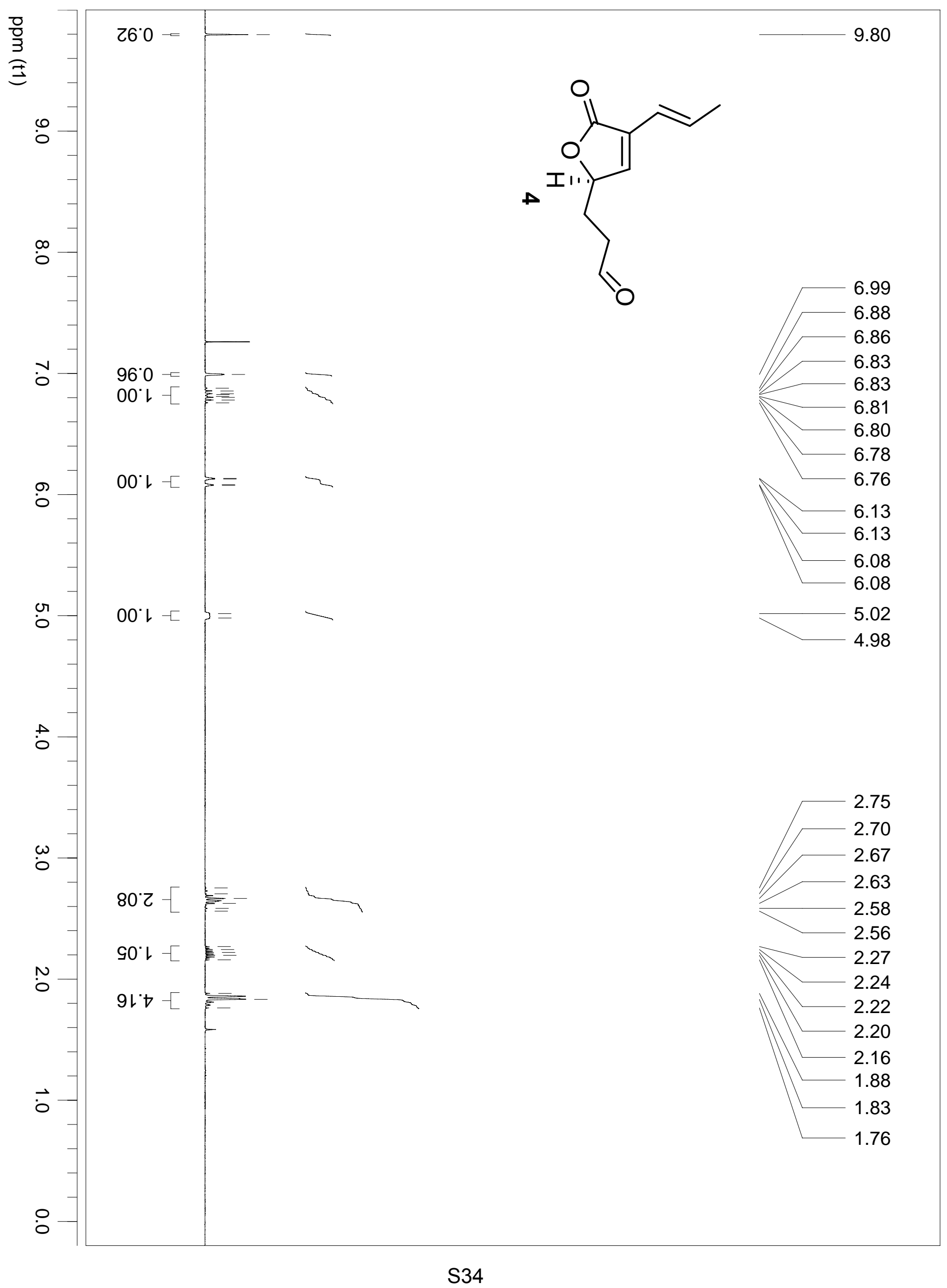




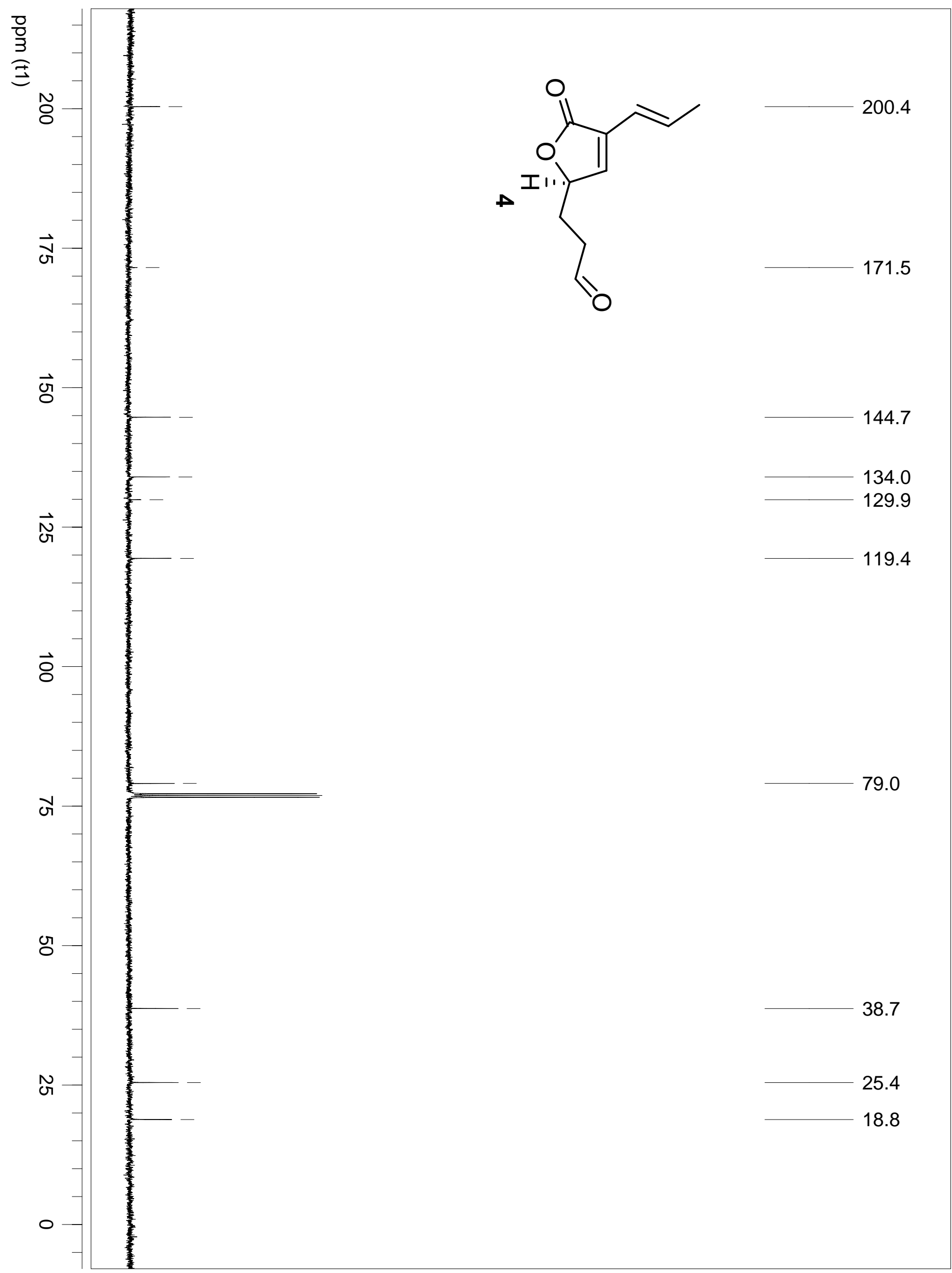




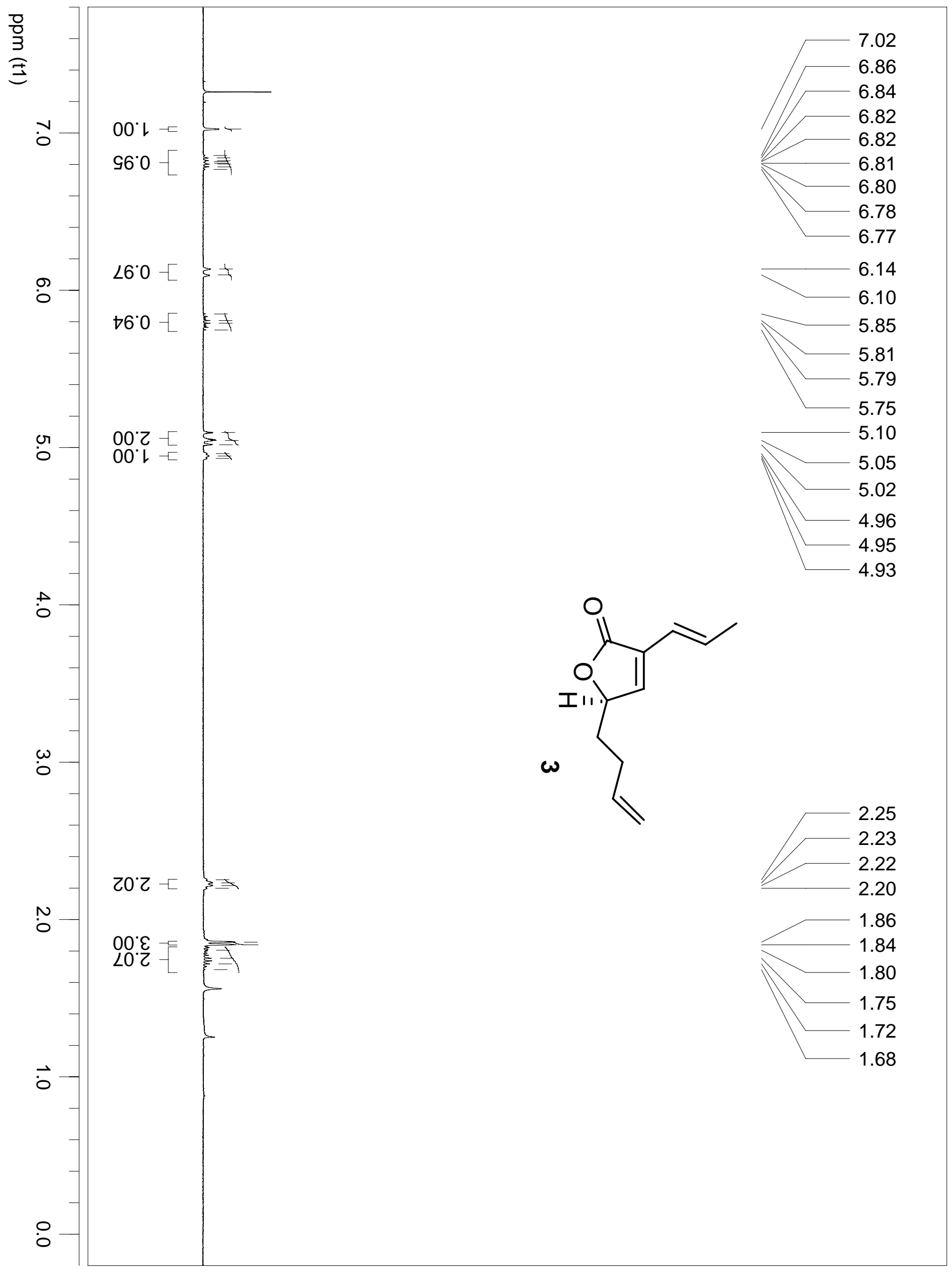




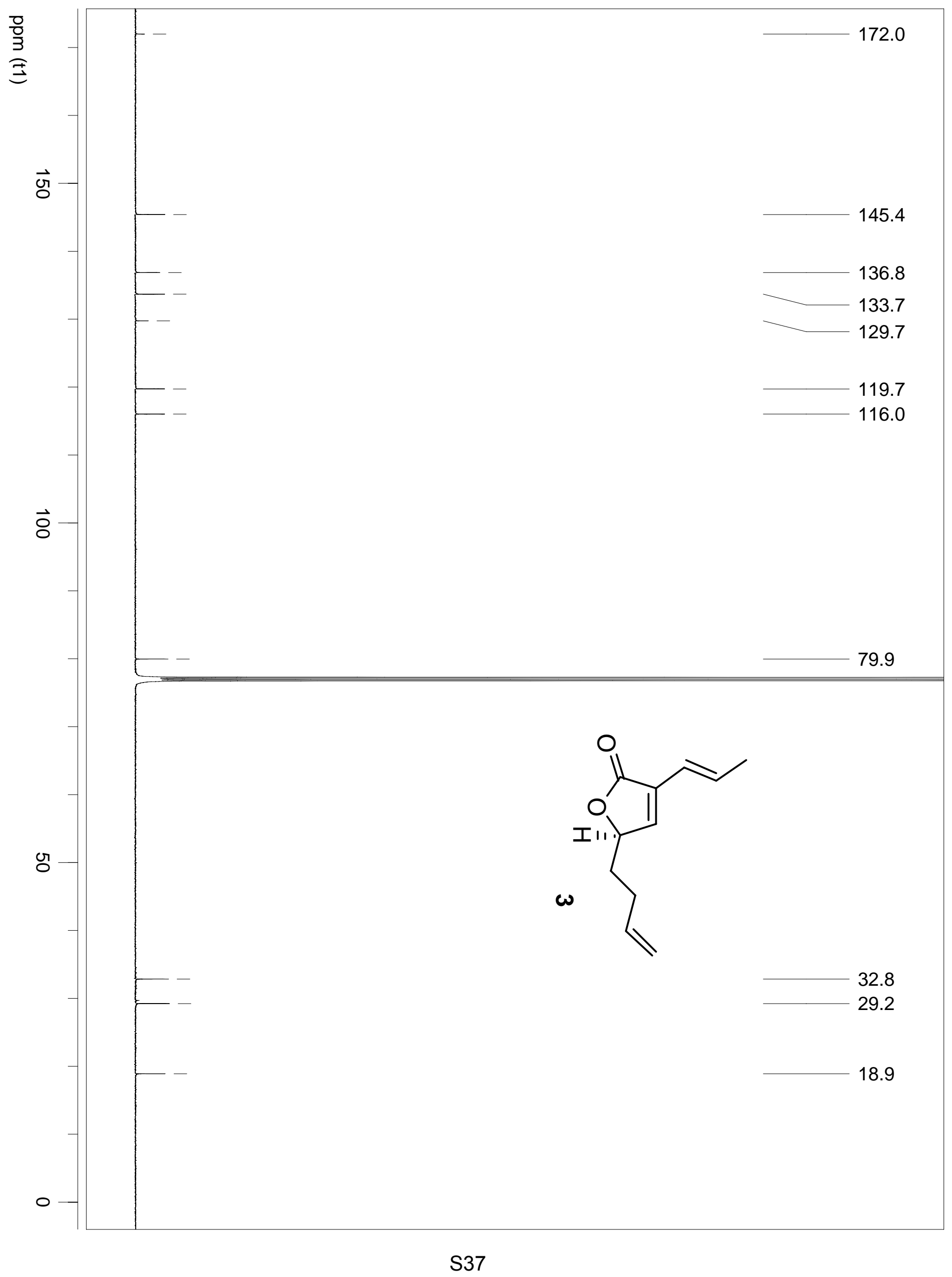




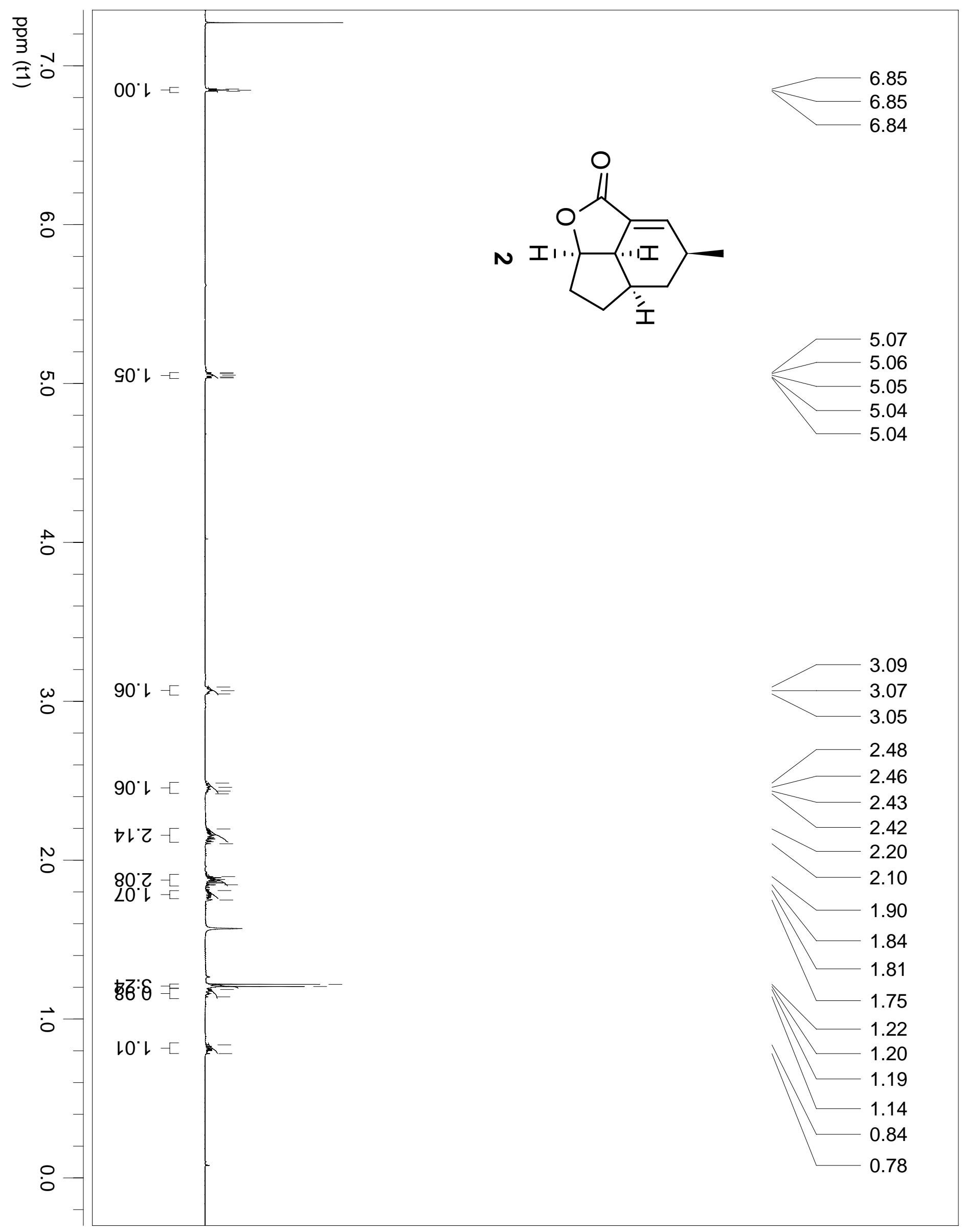




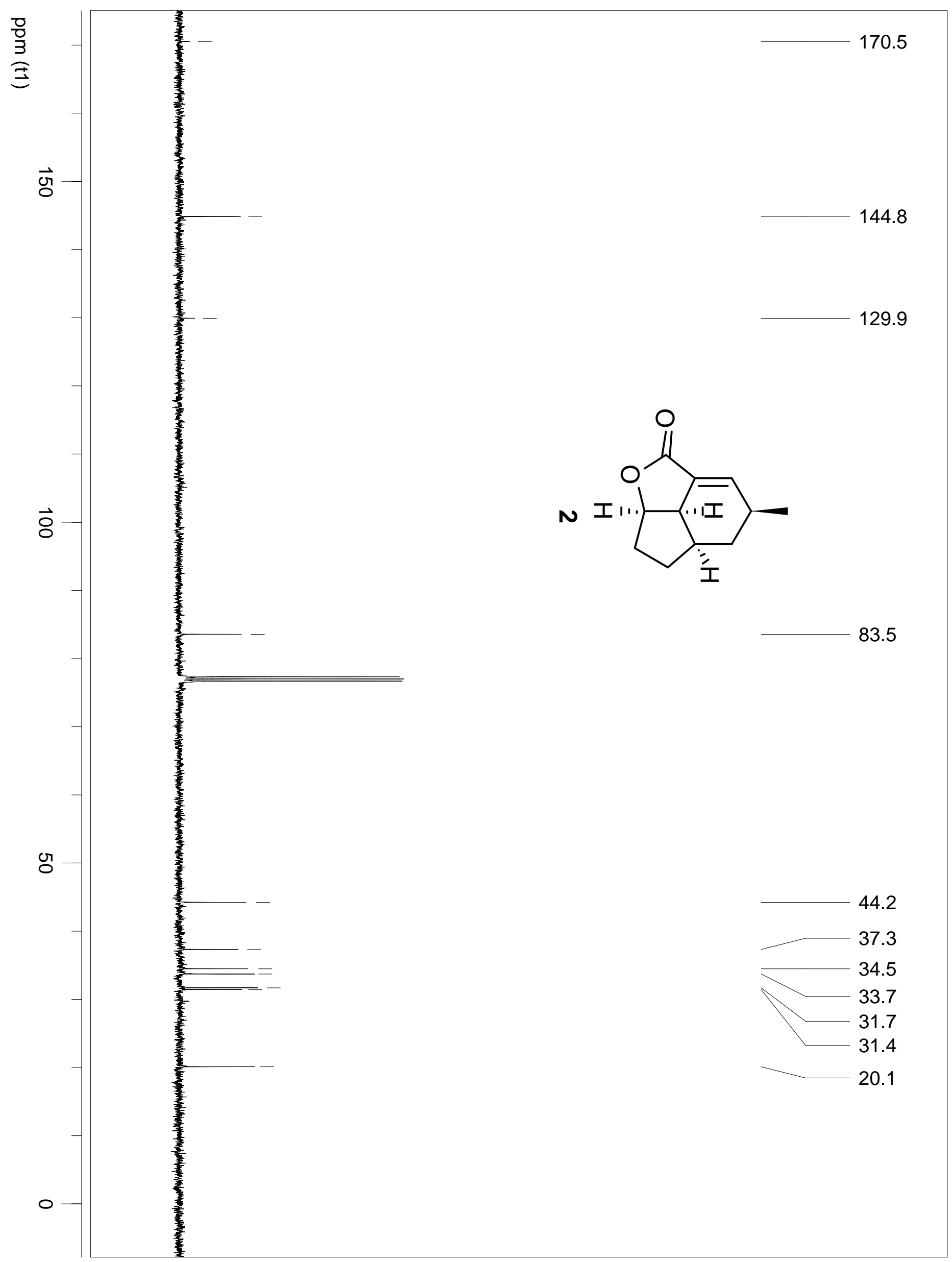

